\title{
The Soybean High Density 'Forrest' by 'Williams 82' SNP-Based Genetic Linkage Map Identifies QTL and Candidate Genes for Seed Isoflavone Content
}

\author{
Dounya Knizia ${ }^{1,2}$, Jiazheng Yuan ${ }^{3}$, Nacer Bellaloui ${ }^{4}$, Tri Vuong ${ }^{5}$, Mariola Usovsky ${ }^{5}$, Qijian Song ${ }^{6}$, \\ Frances Betts $^{3}$, Teresa Register ${ }^{3}$, Earl Williams ${ }^{3}$, Naoufal Lakhssassi ${ }^{1}$, Hamid Mazouz ${ }^{2} \mathbb{D}$, Henry T. Nguyen $^{5} \mathbb{D}^{\mathbb{D}}$, \\ Khalid Meksem ${ }^{1}$ (D), Alemu Mengistu ${ }^{7}$ (D) and My Abdelmajid Kassem ${ }^{3, *(D)}$
}

1 Department of Plant, Soil, and Agricultural Systems, Southern Illinois University, Carbondale, IL 62901, USA; dounya.knizia@siu.edu (D.K.); naoufal.lakhssassi@siu.edu (N.L.); meksem@siu.edu (K.M.)

2 Laboratoire de Biotechnologies \& Valorisation des Bio-Ressources (BioVar), Department de Biology, Faculté des Sciences, Université Moulay Ismail, Meknès 50000, Morocco; H.MAZOUZ@fs-umi.ac.ma

3 Plant Genomics and Biotechnology Laboratory, Department of Biological and Forensic Sciences, Fayetteville State University, Fayetteville, NC 28301, USA; jyuan@uncfsu.edu (J.Y.); fbetts@broncos.uncfsu.edu (F.B.); tregist2@broncos.uncfsu.edu (T.R.); ewilli17@broncos.uncfsu.edu (E.W.)

4 Crop Genetics Research Unit, USDA, Agriculture Research Service, 141 Experiment Station Road, Stoneville, MS 38776, USA; nacer.bellaloui@usda.gov

5 Division of Plant Science and Technology, University of Missouri, Columbia, MO 65211, USA; vuongt@missouri.edu (T.V.); klepadlom@missouri.edu (M.U.); nguyenhenry@missouri.edu (H.T.N.)

check for updates

Citation: Knizia, D.; Yuan, J.; Bellaloui, N.; Vuong, T.; Usovsky, M.; Song, Q.; Betts, F.; Register, T.; Williams, E.; Lakhssassi, N.; et al. The Soybean High Density 'Forrest' by ‘Williams 82' SNP-Based Genetic Linkage Map Identifies QTL and Candidate Genes for Seed Isoflavone Content. Plants 2021, 10, 2029.

https://doi.org/10.3390/plants10102029

Academic Editor: Toyoaki Anai

Received: 23 August 2021

Accepted: 21 September 2021

Published: 27 September 2021

Publisher's Note: MDPI stays neutral with regard to jurisdictional claims in published maps and institutional affiliations.

Copyright: (C) 2021 by the authors. Licensee MDPI, Basel, Switzerland. This article is an open access article distributed under the terms and conditions of the Creative Commons Attribution (CC BY) license (https:/ / creativecommons.org/licenses/by/ $4.0 /)$.
6 Soybean Genomics and Improvement Laboratory, USDA-ARS, Beltsville, MD 20705, USA; qijian.song@usda.gov

7 Crop Genetics Research Unit, USDA, Agricultural Research Service, Jackson, TN 38301, USA; alemu.mengistu@usda.gov

* Correspondence: mkassem@uncfsu.edu

Abstract: Isoflavones are secondary metabolites that are abundant in soybean and other legume seeds providing health and nutrition benefits for both humans and animals. The objectives of this study were to construct a single nucleotide polymorphism (SNP)-based genetic linkage map using the 'Forrest' by 'Williams 82' $(\mathrm{F} \times$ W82) recombinant inbred line (RIL) population $(n=306)$; map quantitative trait loci (QTL) for seed daidzein, genistein, glycitein, and total isoflavone contents in two environments over two years (NC-2018 and IL-2020); identify candidate genes for seed isoflavone. The FXW82 SNP-based map was composed of 2075 SNPs and covered $4029.9 \mathrm{cM}$. A total of 27 QTL that control various seed isoflavone traits have been identified and mapped on chromosomes (Chrs.) 2, 4, 5, 6, 10, 12, 15, 19, and 20 in both NC-2018 (13 QTL) and IL-2020 (14 QTL). The six QTL regions on Chrs. 2, 4, 5, 12, 15, and 19 are novel regions while the other 21 QTL have been identified by other studies using different biparental mapping populations or genome-wide association studies (GWAS). A total of 130 candidate genes involved in isoflavone biosynthetic pathways have been identified on all $20 \mathrm{Chrs}$. And among them 16 have been identified and located within or close to the QTL identified in this study. Moreover, transcripts from four genes (Glyma.10G058200, Glyma.06G143000, Glyma.06G137100, and Glyma.06G137300) were highly abundant in Forrest and Williams 82 seeds. The identified QTL and four candidate genes will be useful in breeding programs to develop soybean cultivars with high beneficial isoflavone contents.

Keywords: soybean; RIL; Forrest; Williams 82; linkage map; isoflavone; daidzein; genistein; glycitein; SNP

\section{Introduction}

Soybean seeds are rich in secondary metabolites beneficial for human and animal consumption including tocopherols, phenolic compounds, saponins, and isoflavones such as genistein, daidzein, and glycitein that showed beneficial health and nutrition effects in animals and humans [1-3]. It is well established that isoflavones reduce menopausal 
symptoms, low density lipoprotein (LDL) cholesterol levels, breast and prostate cancers risks, improve the immune system [4-11], and play an important role in nitrogen fixation and defense against pathogens [12].

Due to these benefits and others, isoflavones, especially genistein, daidzein, and glycitein, have been widely studied during the past decades $[13,14]$ and many studies tried to genetically map quantitative trait loci (QTL) that control seed genistein, daidzein, glycitein, and total isoflavone content as well as their precursors such as daidzin, glycitin, genistin, malonyldaidzin, malonylglycitin, malonylgenistin, etc., using different molecular markers such as AFLPs, RFLPs, SSRs, SNPs [15-27]. For example, using the 'Essex' by 'Forrest' recombinant inbred line (RIL) population $(n=100)$ and $250+$ simple sequence repeat (SSR) markers, 11 QTL that control genistein, daidzein, glycitein, and total isoflavone contents have been identified on Chrs. 1, 3, 7, 8, 11, and 18 [15,16]. Likewise, Liang et al. (2010) used the 'Jindou 23' by 'Huibuzhi' RIL population $(n=474)$ and identified six QTL that control isoflavone contents and mapped them on soybean Chrs. 3, 16, 17, and 18 [18]. In another study, Smallwood et al. (2014) identified 3, 5, 7, and 6 QTL that control seed glycitein, daidzein, genistein, and total isoflavone contents, respectively [20]. Using the 'Zhongdou 27' by 'Jiunong 20' RIL population $(n=130)$ and 194 SSR markers, Han et al. (2015), identified 6, 5, 3, and 7 QTL that control seed glycitein, daidzein, genistein, and total isoflavone contents, respectively [24]. Akond et al. (2015) used the 'Hamilton' by 'Spencer' RIL population ( $n=93$ ), genotyped it with 1502 SNPs, and identified a major QTL that controls both seed daidzein and total isoflavone contents on Chr. 6 and a minor QTL that controls seed glycitein content on Chr. 18 [22]. Recently, the authors of [23] used 'Aokimame' by 'Fukuyutaka' and 'Kumaji-1' by 'Fukuyutaka' RIL populations and identified one QTL that controls malonylgenistin on Chr. 12 and two QTL that control malonylglycitin on Chrs. 11 and 15 [23]. Besides using biparental mapping populations, other researchers used natural populations and genome wide association studies (GWAS) to map QTL that control seed isoflavone contents and identified candidate genes within these QTL regions [28-32].

The objectives of this study were to construct a SNP-based genetic linkage map using the $\mathrm{F} \times$ W82 RIL population $(n=306)$; map quantitative trait loci (QTL) for seed daidzein, genistein, glycitein, and total isoflavone contents in two environments over two years; identify candidate genes involved in soybean seed isoflavone biosynthesis.

\section{Results and Discussion}

\subsection{The SNP-Based Genetic Map}

A total of 5405 SNP markers were generated from the Infinium SNP6K BeadChipsbased genotyping among 306 RILs, from which 2075 polymorphic SNPs were mapped on the 20 soybean chromosomes (Table 1, Figure 1). The F $\times$ W82 genetic map covered $4029.9 \mathrm{cM}$ with an average marker density of $1.94 \mathrm{cM}$ (Table 1). The genetic length ranged from $153.7 \mathrm{cM}$ for Chr. 18 to $308.3 \mathrm{cM}$ for Chr. 2 (Table 1). The polymorphism of SNPs in this RIL population (38.4\%), number of linked SNPs, and map coverage were comparable to other reported SNP-based genetic linkage maps of soybean [33,34]. For example, in Akond et al. (2013) [33], only $27.33 \%$ of SNPs $(1465 / 5361 \times 100)$ have been used to construct the genetic map based on excluding missing data ( 20\%) and heterozygosity (3.99\%). Polymorphic markers between parents (MD96-5722 and Spencer) among the 1465 SNPs used was $44.8 \%(657 / 1465 \times 100)$ [33]. Likewise, in Kassem et al. (2012) [34], polymorphic markers between parents (PI 438489B and Hamilton) among the 1465 SNPs used was $44.2 \%$ $(657 / 1465 \times 100)[34]$. 
Table 1. Distribution of SNP markers and their properties on the Chrs. Of Forrest by Williams 82 recombinant inbred line (RIL) population $(n=306)$.

\begin{tabular}{ccccc}
\hline Chr. & $\begin{array}{c}\text { No. of SNP } \\
\text { Markers }\end{array}$ & Length (cM) & $\begin{array}{c}\text { Average Marker } \\
\text { Density (cM) }\end{array}$ & $\begin{array}{c}\text { Maximum Gap } \\
\text { (cM) }\end{array}$ \\
\hline 1 & 110 & 190.1 & 1.73 & 48.7 \\
2 & 161 & 308.3 & 1.91 & 59.0 \\
3 & 92 & 173.9 & 1.89 & 22.9 \\
4 & 71 & 214.9 & 3.03 & 57.4 \\
5 & 138 & 167.2 & 1.21 & 38.1 \\
6 & 114 & 253.7 & 2.23 & 43.4 \\
7 & 117 & 224.0 & 1.91 & 18.4 \\
8 & 71 & 211.1 & 2.97 & 45.3 \\
9 & 109 & 179.2 & 1.64 & 62.6 \\
10 & 100 & 216.5 & 2.17 & 48.5 \\
11 & 95 & 168.9 & 1.78 & 41.3 \\
12 & 73 & 192.6 & 2.64 & 51.5 \\
13 & 156 & 265.7 & 1.70 & 25.8 \\
14 & 50 & 158.9 & 3.18 & 68.4 \\
15 & 94 & 219.1 & 2.33 & 46.7 \\
16 & 95 & 169.0 & 1.78 & 46.5 \\
17 & 79 & 185.4 & 2.35 & 23.1 \\
18 & 144 & 153.7 & 1.07 & 53.3 \\
19 & 125 & 190.4 & 1.52 & 25.7 \\
20 & 81 & 187.3 & 2.31 & Av. = 43.2 \\
\hline Totals & 2075 & 4029.9 & Av. = 1.94 & \\
\hline
\end{tabular}
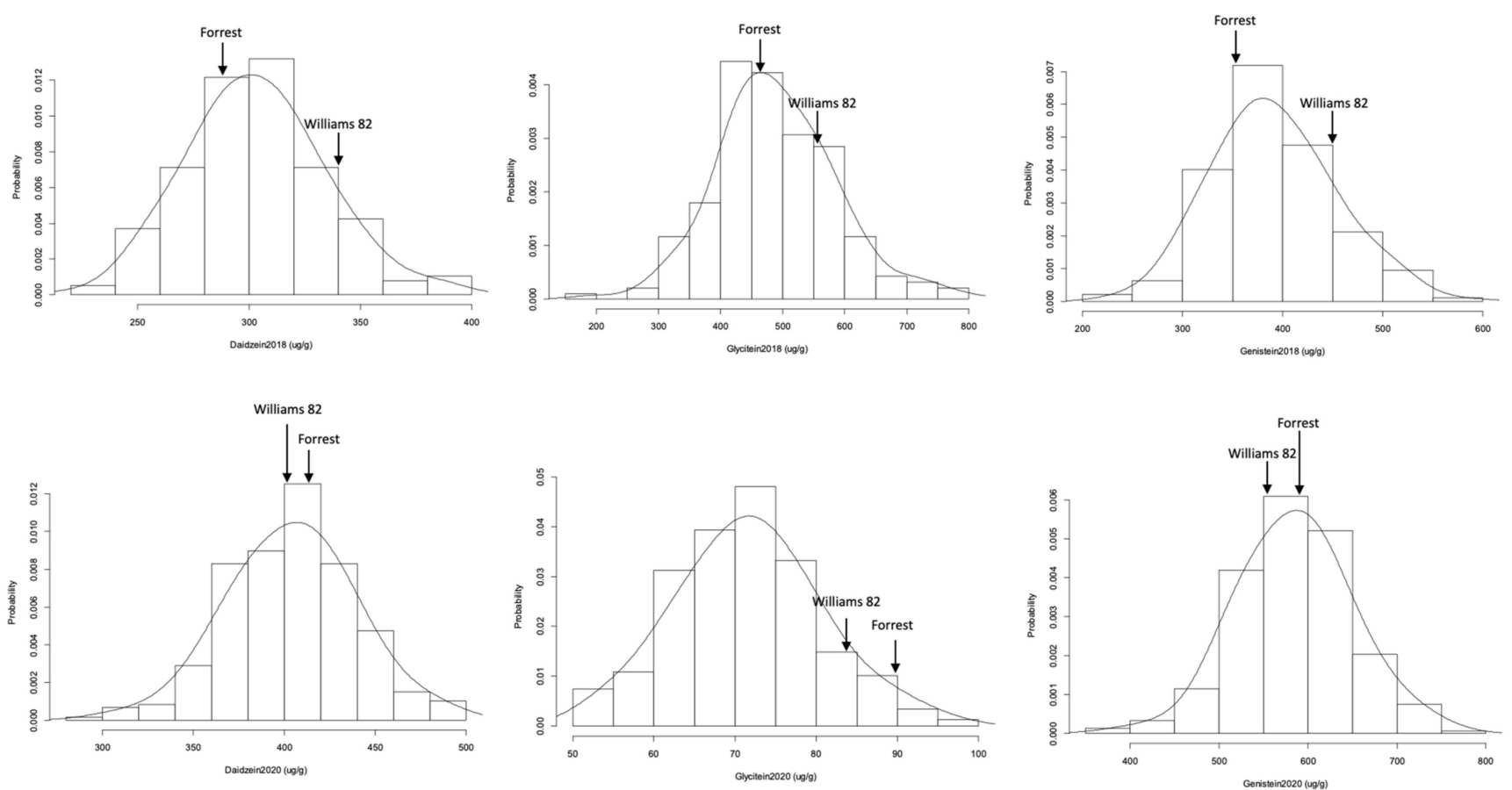

Figure 1. Frequency distribution of seed isoflavone contents ( $\mu \mathrm{g} / \mathrm{g}$ of seed weight) in the FxW82 RIL population. The seed daidzein, genistein, and glycitein contents were assessed in the RILs harvested in Spring Lake, NC (2018) and Carbondale, IL (2020).

\subsection{Isoflavone Contents Frequency Distribution, Heritability, and Correlation}

The seed isoflavone contents were normally distributed in the FxW82 RIL population based on Shapiro-Wilk's method for normality test, even though the positive or negative 
skewness and kurtosis value (>3) were observed in the RIL population (Table 2; Figure 1). The individual component of isoflavone also displayed small ranges of phenotypic variations in the seeds obtained from two geographically diverse field trials (Table 2, Figure 2). Daidzein 2018 in Spring Lake, NC had the highest coefficients of variation (CV) value (19.37\%); however, the CV of this trait in Carbondale, IL (2020) was only $12.59 \%$ suggesting that phenotypic variability among isoflavone contents was impacted by different environmental conditions.

Table 2. Seed isoflavone means, ranges, CVs, skewness, and kurtosis in the FxW82 RIL population evaluated in Spring Lake, NC (2018) and Carbondale, IL (2020). Mean and range values are expressed in $\mu \mathrm{g} / \mathrm{g}$ of seed weight.

\begin{tabular}{cccccccc}
\hline Trait & Mean & Range & CV & SE & Skewness & Kurtosis & $\begin{array}{c}p \text { Value } \\
(\boldsymbol{p}>\mathbf{0 . 0 5})\end{array}$ \\
\hline Daidzein 2018 & 303.22 & 171 & 10.11 & 2.23 & 0.26 & 3.11 & 0.99 \\
Glycitein2018 & 490.47 & 610 & 19.37 & 7.01 & 0.29 & 3.65 & 0.98 \\
Genistein2018 & 391 & 348 & 15.47 & 4.4 & 0.3 & 3.04 & 0.99 \\
Daidzein 2020 & 14.48 & 8.08 & 13.72 & 0.42 & -0.08 & 3.17 & 0.99 \\
Glycitein2020 & 71.79 & 46 & 12.59 & 0.53 & 0.178 & 2.94 & 0.99 \\
Genistein2020 & 584.88 & 383 & 10.94 & 3.73 & -0.02 & 3.31 & 0.99 \\
\hline
\end{tabular}

\section{A. Unassorted correlogram.}

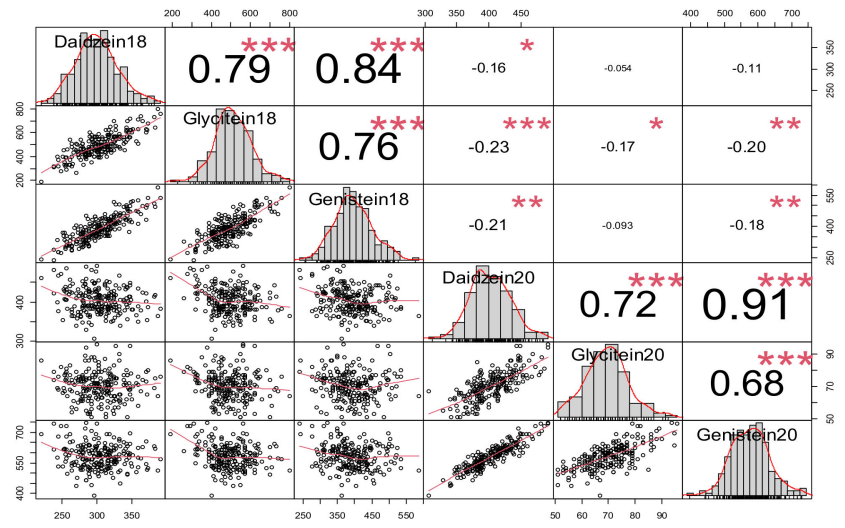

B. Assorted correlogram.

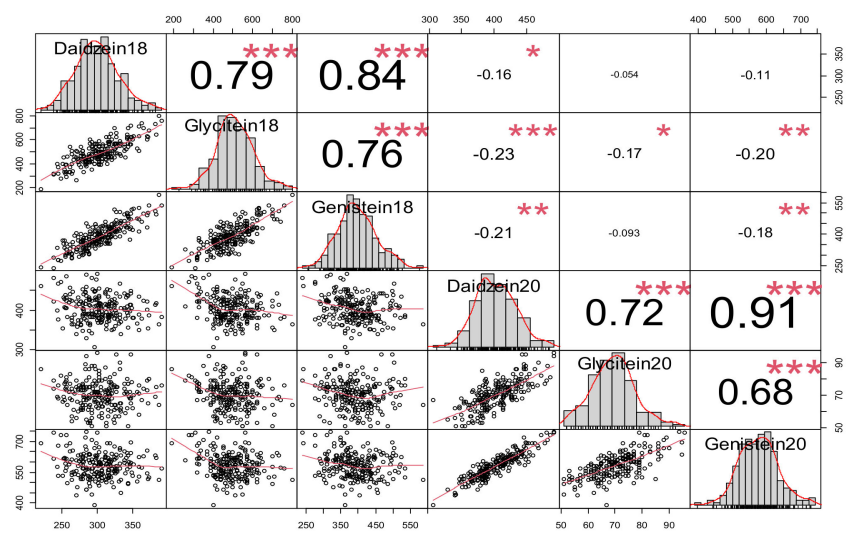

Figure 2. Correlations between daidzein, glycitein, and genistein in the two locations and years: Spring Lake, NC (2018) and Carbondale, IL (2020). (A). Unassorted correlogram, (B). Assorted correlogram. Significance level: ${ }^{*} p<0.05,{ }^{* *} p<0.01$, $* * * p<0.001$.

Azam et al. (2020) [35] reported that the total isoflavones ranged from 745 to $5253.98 \mu \mathrm{g} / \mathrm{g}$, with highest mean of $2689.27 \mu \mathrm{g} / \mathrm{g}$ observed in some regions and up to 2518.91 and $1942.78 \mu \mathrm{g} / \mathrm{g}$ in others due to climatic conditions. Similar results have been reported by other studies [25-27,36-38]. Our results showed over $1000 \mu \mathrm{g} / \mathrm{g}$ and in some cases over $1100 \mu \mathrm{g} / \mathrm{g}$. Therefore, the total concentrations of isoflavones are in the expected range of soybean seed. In addition, there is no premium to be given to growers for soybean seed isoflavone content and no docking is done at the grain elevator for seed isoflavone. Isoflavone concentrations vary depending on the year and environmental growing conditions. Although isoflavones are genetically controlled, environmental conditions including temperature, drought, absence or presence of diseases each year, many other biotic and abiotic factors can significantly affect the contents (by increasing or decreasing) and profile of isoflavone.

The broad sense heritability of percentage dry weight for daidzein, glycitein, and genistein across two different environments over two years appeared to be quite different. Glycitein had the highest heritability (72.4\%) and the values for both daidzein and 
genistein were $42.8 \%$ and $42.5 \%$, respectively, which displayed a similar fashion. The lower heritability of daidzein and genistein contents suggested that some portion of phenotypic variation was still not detected by the mapped QTL due to the complexity of these traits. The genotype-environment interactions still played a significant role in the molecular formation of daidzein and genistein molecules in soybean seeds based on our two-way ANOVA analysis because the $\sigma_{\mathrm{GE} 2}$ is relatively high compared to that of glycitein (data not shown). It will certainly impact future breeding strategies for trait improvement based on the data we presented on these traits.

We used type I sum of squares (ANOVA (model)) function in R program to obtain the Sum Sq and Mean Sq and calculated $\sigma_{\mathrm{G}}{ }^{2}$ and $\sigma_{\mathrm{GE}}{ }^{2}$ for each trait (Table 3). However, $\sigma_{\mathrm{e}}{ }^{2}$ was 0 due to limited replicates. In this study, we only had three technical replicates due to cost effect of this student-centered project, but these replicates could only be considered as one biological replicate and hence, $\mathrm{F}$ value and probability could not be generated (Table 3 ). The FxW82 RIL population derived from two parental cultivars with different maturity groups (MGs). Forrest belongs to MG5-6 and Williams 82 to MG2-3 suggesting that the locations may play an important role on major agronomic traits including seed isoflavone. Based on our data (Figure 2), glycitein showed less correlation with daidzein and genistein which may indicate that its production may be less impacted by environment. Furthermore, Fayetteville, NC is a subtropic favorable weather for MG6-7 soybeans while Carbondale, IL is the favorable weather for MG 4-5. Therefore, further studies of the seed isoflavone in the FxW82 RIL population in different environments would be beneficial.

Table 3. Two-way ANOVA results for daidzein, genistein, and glycitein.

\begin{tabular}{|c|c|c|c|c|}
\hline \multicolumn{5}{|c|}{ Response: Daidzein } \\
\hline & Df & Sum Sq & Mean Seq & $\mathrm{H}^{2}$ \\
\hline Line & 301 & 541,800 & 1800 & 0.428 \\
\hline Year & 1 & 974,711 & 974,711 & \\
\hline Line: Year & 181 & 186,226 & 1029 & \\
\hline Residuals & 0 & 0 & NA & \\
\hline \multicolumn{5}{|c|}{ Response: Glycitein } \\
\hline & Df & Sum Sq & Mean Seq & $\mathrm{H}^{2}$ \\
\hline Line & 301 & $5,086,274$ & 16,898 & 0.724 \\
\hline Year & 1 & $16,033,506$ & $16,033,506$ & \\
\hline Line: Year & 181 & 843,473 & 4660 & \\
\hline Residuals & 0 & 0 & NA & \\
\hline \multicolumn{5}{|c|}{ Response: Genistein } \\
\hline & Df & Sum Sq & Mean Seq & $\mathrm{H}^{2}$ \\
\hline Line & 301 & $1,922,339$ & 6387 & 0.425 \\
\hline Year & 1 & $3,630,207$ & $3,630,207$ & \\
\hline Line: Year & 181 & 668,735 & 3695 & \\
\hline Residuals & 0 & 0 & NA & \\
\hline
\end{tabular}

The correlogram demonstrates a novel correlation among these assessed traits (Figure 2). Based on the unassorted data (all lines were included), each of the isoflavone components was positively correlated with the other sister isoflavones $(p<0.001)$ from the same geographical location but negatively correlated with the isoflavones from the other location inferring that the production of these isoflavones has been strongly impacted both by genotype and environmental conditions. The assorted data (lines tested in both locations) showed similar positivity, but the level of negative correlation was low (Figure 2). To the best of our knowledge, this observation has not been described in other studies. 


\subsection{Seed Isoflavone Contents QTL}

Both interval mapping (IM) and composite interval mapping (CIM) methods of WinQTL Cartographer 2.5 [39] were used to identify QTL for seed daidzein, genistein, glycitein, and total isoflavone contents in the present RIL population. A total of 27 QTL that control seed isoflavone contents have been identified in this population in both NC-2018 (13 QTL) and IL-2020 (14 QTL) (Table 4, Figure 3 and Figure S1).

Table 4. QTL that control seed isoflavone (daidzein, genistein, and glycitein) contents in two environments over two years (2A. 2018 and 2B. 2020). The two environments are Spring Lake, NC (2018) (2A) and Carbondale, IL (2020) (2B). Only QTL with LOD scores > 2.0 and identified by composite interval mapping (CIM) method of QTL Cartographer (Wang et al., 2012) are reported.

\begin{tabular}{|c|c|c|c|c|c|c|c|c|}
\hline \multicolumn{9}{|c|}{ 2A. QTL Identified in Spring Lake, NC (2018) } \\
\hline Trait & QTL & Chr. & Marker & $\begin{array}{c}\text { Interval } \\
\text { (cM) }\end{array}$ & LOD & $R^{2}(\%)$ & $\begin{array}{l}\text { Additive } \\
\text { Effect }\end{array}$ & Environment \\
\hline Daidzein & $q D A I D 01$ & 5 & Gm05_1705841 & 160.41 & 2.01 & 6.07 & -7.61 & Spring Lake, NC \\
\hline Daidzein & & 5 & Gm05_9012813 & 166.51 & 2.12 & 4.18 & -6.35 & Spring Lake, NC \\
\hline Daidzein & & 5 & Gm05_9097414 & 166.71 & 2.19 & 4.32 & -6.46 & Spring Lake, NC \\
\hline Daidzein & & 5 & Gm05_8916450 & 166.81 & 2.11 & 4.16 & -6.34 & Spring Lake, NC \\
\hline Genistein & qGEN03 & 5 & Gm05_1705841 & 152.41 & 2.06 & 9.37 & -18.72 & Spring Lake, NC \\
\hline Genistein & & 5 & Gm05_9012813 & 166.51 & 2.27 & 4.22 & -12.53 & Spring Lake, NC \\
\hline Genistein & & 5 & Gm05_9097414 & 166.71 & 2.36 & 4.39 & -12.79 & Spring Lake, NC \\
\hline Genistein & & 5 & Gm05_8916450 & 166.81 & 2.28 & 4.25 & -12.57 & Spring Lake, NC \\
\hline Glycitein & qGLY02 & 5 & Gm05_1705841 & 146.41 & 2.01 & 9.07 & -29.89 & Spring Lake, NC \\
\hline Genistein & $q G E N 01$ & 6 & Gm06_5014399 & 64.41 & 2.58 & 8.52 & 21.15 & Spring Lake, NC \\
\hline Daidzein & $q D A I D 02$ & 6 & Gm06_5014399 & 62.41 & 2.06 & 7.55 & 10.35 & Spring Lake, NC \\
\hline Daidzein & & 6 & Gm06_3941524 & 78.21 & 2.02 & 7.24 & 8.67 & Spring Lake, NC \\
\hline Genistein & $q G E N 04$ & 6 & Gm06_5014399 & 60.41 & 2.26 & 9.06 & 22.94 & Spring Lake, NC \\
\hline Genistein & & 6 & Gm06_3941524 & 70.21 & 2.11 & 3.98 & 13.86 & Spring Lake, NC \\
\hline Genistein & qGEN02 & 12 & Gm12_915327 & 179.21 & 2.56 & 4.8 & -16.87 & Spring Lake, NC \\
\hline Genistein & & 12 & Gm12_1064727 & 179.41 & 2.58 & 4.85 & -16.93 & Spring Lake, NC \\
\hline Genistein & & 12 & Gm12_1229101 & 179.71 & 2.95 & 5.51 & -17.79 & Spring Lake, NC \\
\hline Genistein & & 12 & Gm12_1374970 & 179.91 & 2.95 & 5.5 & -17.78 & Spring Lake, NC \\
\hline Genistein & & 12 & Gm12_1433336 & 180.61 & 2.85 & 5.33 & -17.4 & Spring Lake, NC \\
\hline Glycitein & qGLY01 & 12 & Gm12_553862 & 177.31 & 2.76 & 5.82 & -26.95 & Spring Lake, NC \\
\hline Glycitein & & 12 & Gm12_915327 & 179.21 & 2.58 & 4.79 & -27.05 & Spring Lake, NC \\
\hline Glycitein & & 12 & Gm12_1064727 & 179.41 & 2.59 & 4.82 & -27.12 & Spring Lake, NC \\
\hline Glycitein & & 12 & Gm12_1229101 & 179.71 & 2.83 & 5.23 & -27.76 & Spring Lake, NC \\
\hline Glycitein & & 12 & Gm12_1374970 & 179.91 & 2.83 & 5.23 & -27.76 & Spring Lake, NC \\
\hline Glycitein & & 12 & Gm12_1433336 & 180.61 & 2.7 & 5.02 & -27.03 & Spring Lake, NC \\
\hline Genistein & qGEN05 & 12 & Gm12_553862 & 171.31 & 2.03 & 5.74 & -15.18 & Spring Lake, NC \\
\hline Genistein & & 12 & Gm12_975837 & 178.71 & 2.47 & 4.64 & -16.47 & Spring Lake, NC \\
\hline Genistein & & 12 & Gm12_1632399 & 181.31 & 2.32 & 4.38 & -15.76 & Spring Lake, NC \\
\hline Glycitein & qGLY03 & 12 & Gm12_553862 & 169.31 & 2.22 & 5.63 & -23.71 & Spring Lake, NC \\
\hline Glycitein & & 12 & Gm12_975837 & 178.71 & 2.46 & 4.58 & -26.29 & Spring Lake, NC \\
\hline Glycitein & & 12 & Gm12_1632399 & 181.31 & 2.2 & 4.1 & -24.52 & Spring Lake, NC \\
\hline Daidzein & qDAID03 & 19 & Gm19_4552537 & 109.51 & 2.14 & 4.17 & 6.92 & Spring Lake, NC \\
\hline Glycitein & qGLY04 & 19 & Gm19_3010363 & 35.31 & 2.04 & 3.83 & 19.46 & Spring Lake, NC \\
\hline Genistein & qGEN06 & 20 & Gm20_4657454 & 0.01 & 2.03 & 3.75 & 11.84 & Spring Lake, NC \\
\hline
\end{tabular}


Table 4. Cont.

\begin{tabular}{|c|c|c|c|c|c|c|c|c|}
\hline \multicolumn{9}{|c|}{ 2B. QTL Identified in Carbondale, IL (2020) } \\
\hline Trait & QTL & Chr. & Marker & $\begin{array}{l}\text { Interval } \\
\text { (cM) }\end{array}$ & LOD & R2 (\%) & $\begin{array}{l}\text { Additive } \\
\text { Effect }\end{array}$ & Environment \\
\hline Daidzein & qDAID03 & 2 & Gm02_2282900 & 24.01 & 2.01 & 10.61 & -11.55 & Carbondale, IL \\
\hline Genistein & qGEN04 & 4 & Gm04_4461164 & 190.31 & 2.26 & 3.96 & -12.97 & Carbondale, IL \\
\hline Genistein & $q G E N 01$ & 10 & Gm10_4670275 & 130.81 & 2.6 & 3.52 & -12.38 & Carbondale, IL \\
\hline Genistein & & 10 & Gm10_4035277 & 130.91 & 2.61 & 3.53 & -12.39 & Carbondale, IL \\
\hline Daidzein & $q D A I D 04$ & 10 & Gm10_4670275 & 130.81 & 2.18 & 2.97 & -6.11 & Carbondale, IL \\
\hline Daidzein & & 10 & Gm10_4035277 & 130.91 & 2.19 & 2.97 & -6.12 & Carbondale, IL \\
\hline Genistein & qGEN05 & 10 & Gm10_4670275 & 128.81 & 2.15 & 3.2 & -11.75 & Carbondale, IL \\
\hline Genistein & & 10 & Gm10_4035277 & 132.91 & 2.37 & 3.39 & -12.11 & Carbondale, IL \\
\hline Daidzein & qDAID01 & 12 & Gm12_9193994 & 53.21 & 2.56 & 4.07 & 7.18 & Carbondale, IL \\
\hline Daidzein & & 12 & Gm12_1430950 & 61.71 & 3.99 & 5.61 & 8.49 & Carbondale, IL \\
\hline Daidzein & & 12 & Gm12_1423120 & 62.31 & 4.12 & 5.78 & 8.62 & Carbondale, IL \\
\hline Daidzein & & 12 & Gm12_1539402 & 63.01 & 4.53 & 6.34 & 9.01 & Carbondale, IL \\
\hline Daidzein & & 12 & Gm12_1678702 & 63.11 & 4.59 & 6.45 & 9.1 & Carbondale, IL \\
\hline Daidzein & & 12 & Gm12_3052701 & 64.11 & 4.89 & 6.82 & 9.42 & Carbondale, IL \\
\hline Daidzein & & 12 & Gm12_2097199 & 64.41 & 4.4 & 6.18 & 8.97 & Carbondale, IL \\
\hline Daidzein & & 12 & Gm12_2432082 & 65.31 & 4.26 & 5.97 & 8.77 & Carbondale, IL \\
\hline Daidzein & & 12 & Gm12_1547239 & 65.51 & 3.77 & 5.31 & 8.27 & Carbondale, IL \\
\hline Daidzein & & 12 & Gm12_1428801 & 65.91 & 3.36 & 4.75 & 7.87 & Carbondale, IL \\
\hline Genistein & qGEN02 & 12 & Gm12_9193994 & 55.21 & 2.5 & 4.24 & 13.58 & Carbondale, IL \\
\hline Genistein & & 12 & Gm12_1430950 & 61.71 & 3.78 & 5.28 & 15.31 & Carbondale, IL \\
\hline Genistein & & 12 & Gm12_1423120 & 62.31 & 3.76 & 5.25 & 15.2 & Carbondale, IL \\
\hline Genistein & & 12 & Gm12_1539402 & 63.01 & 4.39 & 6.1 & 16.4 & Carbondale, IL \\
\hline Genistein & & 12 & Gm12_1678702 & 63.11 & 4.38 & 6.1 & 16.4 & Carbondale, IL \\
\hline Genistein & & 12 & Gm12_3052701 & 64.11 & 4.66 & 6.46 & 16.99 & Carbondale, IL \\
\hline Genistein & & 12 & Gm12_2097199 & 64.41 & 4.05 & 5.65 & 15.64 & Carbondale, IL \\
\hline Genistein & & 12 & Gm12_2432082 & 65.31 & 3.83 & 5.35 & 15.25 & Carbondale, IL \\
\hline Genistein & & 12 & Gm12_1547239 & 65.51 & 3.38 & 4.74 & 14.39 & Carbondale, IL \\
\hline Genistein & & 12 & Gm12_1428801 & 65.91 & 3.16 & 4.44 & 13.96 & Carbondale, IL \\
\hline Daidzein & qDAID05 & 12 & Gm12_9193994 & 51.21 & 2.03 & 2.91 & 6.05 & Carbondale, IL \\
\hline Daidzein & & 12 & Gm12_1428801 & 73.91 & 2.31 & 4.8 & 7.83 & Carbondale, IL \\
\hline Genistein & qGEN06 & 12 & Gm12_1428801 & 71.91 & 2.37 & 4.4 & 13.77 & Carbondale, IL \\
\hline Glycitein & $q G L Y 01$ & 15 & Gm15_756303 & 212.31 & 2.07 & 2.86 & -1.53 & Carbondale, IL \\
\hline Glycitein & & 15 & Gm15_2072075 & 218.41 & 2.24 & 3.1 & -1.6 & Carbondale, IL \\
\hline Glycitein & & 15 & Gm15_2021199 & 218.81 & 2.06 & 2.84 & -1.53 & Carbondale, IL \\
\hline Daidzein & $q D A I D 02$ & 20 & Gm20_3804081 & 70.31 & 2.55 & 6.13 & -8.81 & Carbondale, IL \\
\hline Genistein & qGEN03 & 20 & Gm20_3804081 & 68.31 & 2.65 & 6.72 & -16.81 & Carbondale, IL \\
\hline Daidzein & $q D A I D 06$ & 20 & Gm20_3804081 & 66.31 & 2.22 & 5.58 & -8.41 & Carbondale, IL \\
\hline Genistein & qGEN07 & 20 & Gm20_3804081 & 64.31 & 2 & 5.08 & -14.62 & Carbondale, IL \\
\hline Genistein & & 20 & Gm20_3424023 & 80.01 & 2.44 & 3.3 & -11.8 & Carbondale, IL \\
\hline Genistein & & 20 & Gm20_3418121 & 80.51 & 2.1 & 2.85 & -10.96 & Carbondale, IL \\
\hline
\end{tabular}




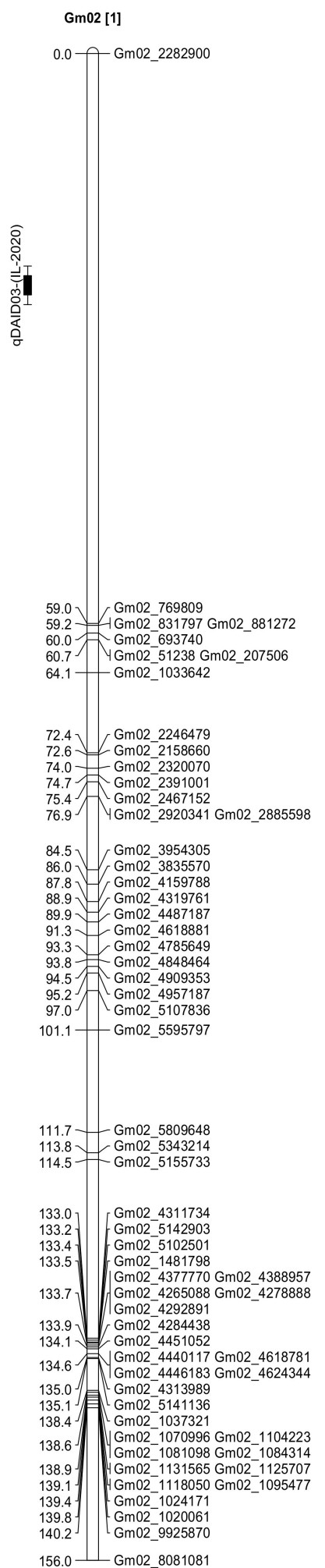

Gm02 [2]

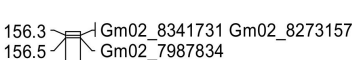

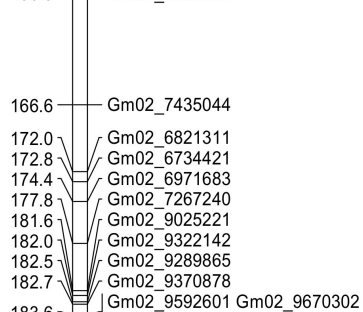

183.6- $\mathrm{Gm02}_{-} 9592601$

184.2 $\bigwedge_{\text {Gm02 }} 9873369$

184.7 Gm02_9795247

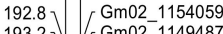

- Gm02_ 1149487

Gm02 1162889

197.3 Gm02_1207820

98.0 $\mathrm{Gm02} 1216868$

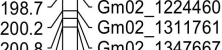

201.5] Gm02_1355215

203.6 Gm02_ 1373746

207.7 $\mathrm{Gm02} 1412741$

08.7 Gm02_1421548

Gm02_1434542

229.4] [ Gm02_3003645

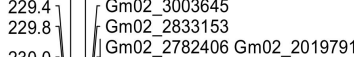

230.0 . Gm02 2782406

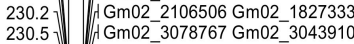

232.2 / Gm02_3547150 Gm02_3666668 $232.6=\mid \begin{aligned} & \text { Gm02 } \\ & \text { Gm02 } 3954819 \text { Gm02 } 3744195 \mathrm{Gm02} 3826152\end{aligned}$

232.6

233.3 㲘 Gm02_3993105

234.3 Gm02_4001947

234.8] $4 \mathrm{Gm02} 4102188$

235.2. $\mathrm{Gm02}-4073086$

Gm02 4140085

Gm02_4158397

Gm02 4178834

37.0 $4 \mathrm{Gm02} 4186050 \mathrm{Gm02} 4193112$

$237.5-G m 024197940$

238.8 Gm02 4207385

239.9 Gm02_4220473

242.0 - Gm02-4240487

242.8 ] Gm02_4273764

252.7 . Gm02-4403315

254.2 Gm02 4419067 Gm02 4415663

254.6. $\mathrm{Gm02}-4411437$

$258.0]\left[\begin{array}{l}\mathrm{Gm02}-4425623 \\ \mathrm{Gm02} 4477896\end{array}\right.$

274.4 Gm02_4663092

275.7 - Gm02_467783

277.0 $\cup(G m 02-4685522$

$277.4 \widehat{A ~}_{\mathrm{GmO2}}-4706497$

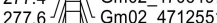

279.3 $]-G m 02-4726820$

280.1 Gm02 4731435

282.2 $4 \mathrm{Gm02} 4752873 \mathrm{Gm02} 4751517$

$282.7-G m 02-4779030$

$286.4 \quad$ Gm02_4838212

295.8 $\quad \mathrm{Gm02} 4938821$

297.3] Gm02_4961612

298.0 Gm02_4977619

$300.9][G m 02-4998671$

$301.6]-(G m 02-5017503$

304.0 $)$ ( A Gm02_5038341 Gm02_5035834

305.2 Gm02 5054614

305.7 Y Gm02_5070691 Gm02 5071055

306.6 Gm02 5093657

$\left.\begin{array}{l}307.8 \\ 308.3\end{array}\right]$ Gm02_5115475
Gm04 [1]

0.0 年 Gm04_4885662

9919 Gm04_4628043

$0.5\left[\begin{array}{l}\mathrm{Gm} 04-4651712 \mathrm{GmO4}-4577396 \\ \mathrm{Gm} 04-4824411\end{array}\right.$

1.0 Gm04_2953580

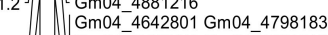
Gm04_4795771 Gm04_4781513

(a)

6.5 $\mathrm{I}_{\mathrm{Gm} 04-7655021}^{\mathrm{Gm}} \mathrm{G}$

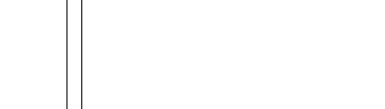

131.2 Gm04_8004029

132.6 131.6 Gm04_8065917

3.9-Gm04 547510

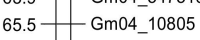

68.3-Gm04_717646

$75.0-$ Gm04_1621110

79.8 Gm04 2237308

80.3 - Gm04_226864

83.2 - Gm04-2681971

83.2 $>$ Gm04-2681971

Gm04-6064896

120.2 Gm04 6643461

121.8 $\mathrm{Gm04}-7052221$

12.8 $\mathrm{Gm04}-7213250$

123.8 Gm04-7213250

-8317828

Gm04_4081102

149.6. Gm04 412493

150.3 Gm04-4132821

$151.1]$ Gm04_4215393

151.5 - Gm04_4153779

(156.8

56.8 $]$ [ Gm04-4295137

58.7 Gm04 4305966 Gm04 4305849

$159.2=$ Gm04_4315787

$162.2][\mathrm{Gm04} 4350583$

162.6 Gm04-4358414

( $\mathrm{Gm04}^{-4373833}$

$165.3 .404-4388529$

6 - Gm04 438008

Gm04 439668

66.7 Gm04_4404611

(67.6 Gm04_4411449

(68.0

69.3 Gm04-4429082

Gm04_44337

170.4 $\left[\begin{array}{l}\mathrm{G} m 04-4438393 \\ \mathrm{Gm04} 4461164\end{array}\right.$

Gm04_4461164

106.9

Gm04_517218

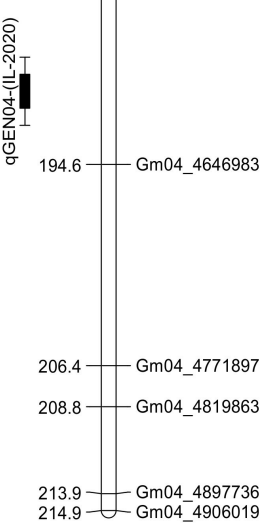

Figure 3. Cont. 

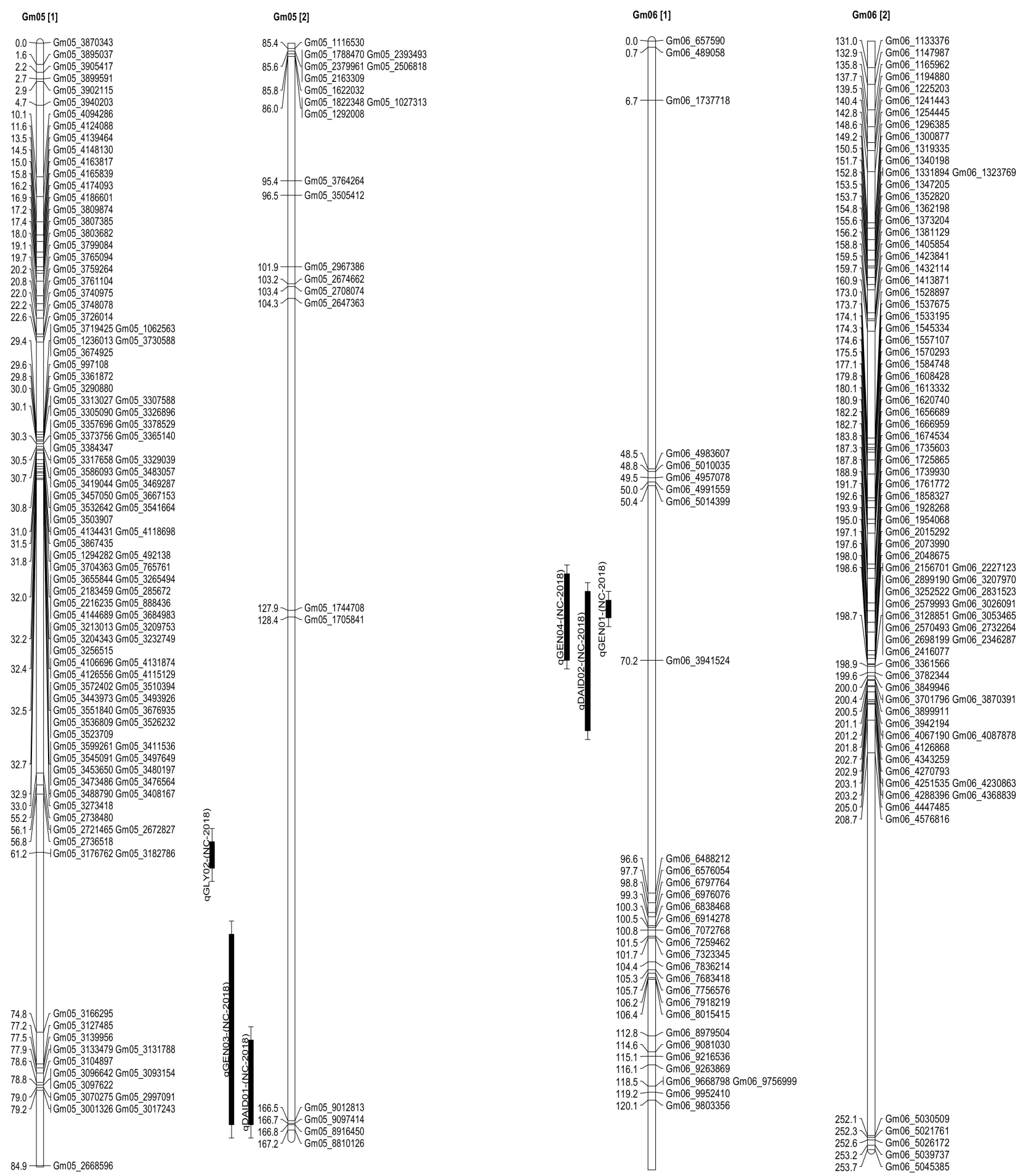

Figure 3. Cont. 


\section{Gm10 [1]}

$0.0-\mathrm{Gm} 104999574$

$4.8]-[\mathrm{Gm10} 4963066$

5.5] $[\mathrm{Gm10}-4955930$

$6.3]=[\mathrm{Gm} 10-4944758$

. Gm10_4888012 Gm10_489665

$=1 \mathrm{Gm} 10^{-} 4903450$

9.2 才 Gm10-4864349 Gm10_4858613

11.1 - Gm10 4842697

11.1 政 $\mathrm{Gm} 10$-4842697

12.1] $[\mathrm{Gm10}-4833115$

$12.7]$ Gm10_4811140

13.0 $\mathrm{Gm10} 4794663$

13.2. Gm10-4798433

14.7 [ Gm10 4777478

$23.0-G m 10 \_4678861$

27.6 [Gm10_4635111

8.6. $]\left[\begin{array}{l}\mathrm{Gm} 10-4635111 \\ \mathrm{Gm} 10^{-460698}\end{array}\right.$

$28.4=\mathrm{Gm} 10-461775$

28.7 Gm10_4626943

$29.0-G m 10-4611925$

30.3 Gm10_4589013

31.4 $]$ Gm10_4567053

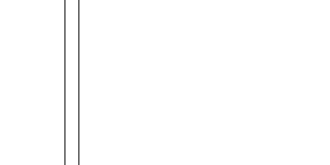

46.2] Gm10 4422765

$47.9]-\mathrm{Gm10} 4378326$

$48.8=-\mathrm{Gm}_{10}{ }^{-4361205}$

$49.5] \mathrm{Gm}$

$49.7]\left[\begin{array}{ll}\mathrm{Gm} 10 \\ -4384037\end{array}\right.$

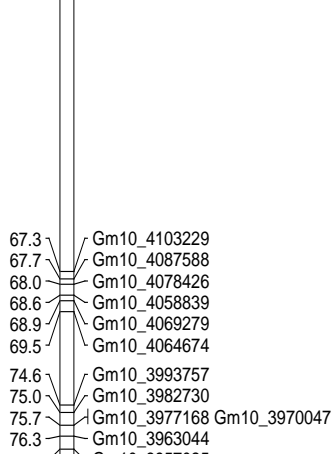

$77.0-\mathrm{Gm10} 3957025$

79.2 . $\mathrm{Gm} 10^{-} 3943637$

79.4 ${ }_{\text {Gm10 }} 3935014$

84.4 Gm10_3890052

$85.1-\mathrm{Gm10}^{-3882257}$

85.7 - $\mathrm{Gm10}$-3875166

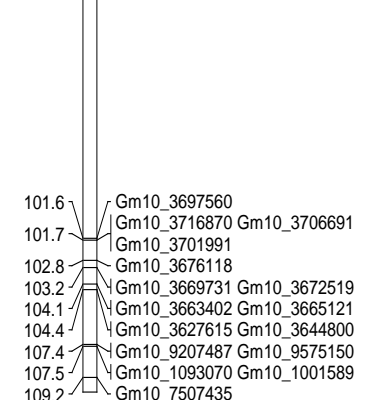

Gm10 [2]

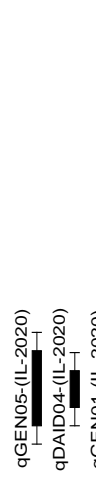

$110.7-\square$ Gm10_6663940

$112.5-G m 10 \_6524119$

$115.3-G m 105922076$

115.8 Gm10_5556984

118.6-Gm10_5295541

122.8-Gm10_4670275

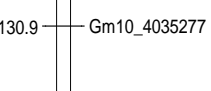

135.7-Gm10_3465857

138.4 Gm10_3235061

- Gm10 2376787

140.4 Gm10 3066211

143.2 $\mathrm{Gm} 10^{-2} 2714130$

$146.6-$ Gm10_2482570

149.2-Gm10_2240113

156.6- Gm10_1667248

156.6-Gm10_1667248

161.8 Gm10_1426801

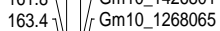

164.4 $]$. Gm10_1232205

165.3] Gm10-981062

165.5] Gm10-831916

$165.8=-\mathrm{Gm10} 925972$

166.9 $\mathrm{Gm10}-214458$

167.3 Gm10_121493

$167.8] \llbracket$ Gm10-754804

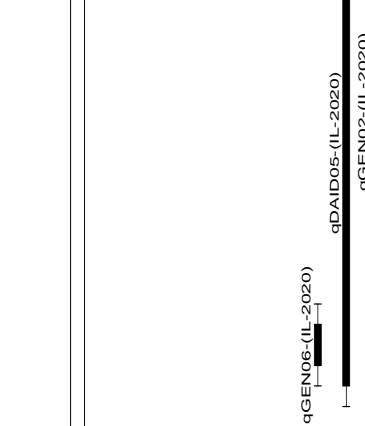

I

51.2-IGm12_9153986 Gm12_9193994

32.8-1Gm12_3442123 Gm12_3438049

33.5 - $\mathrm{Gm} 12$ - 3421704

$36.0-G m 12$ _3389214

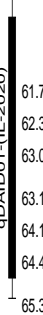

Gm12_1469459 Gm12_1435257 Gm12 1423120

Gm12__1551411 Gm12_1545161

Gm12 1792675 Gm12 2427183

GIII2-1678702

IGm12_1995885 Gm12_2951774

Gm12 209719

Gm12_2238523 Gm12_1701326

655 . Gm12 $1587749 \mathrm{Gm} 121541927$

5.9 ${ }_{G m 12}$ _1428801

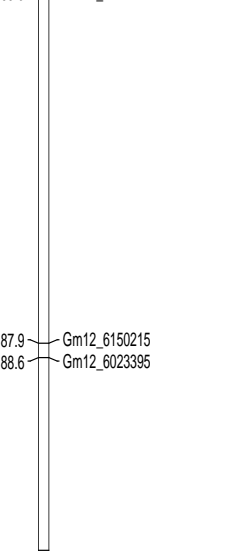

Gm12 [2]

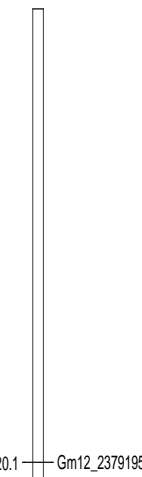

36.4 —GM12 3309895

$139.0]$ Gm12 322542

1300 -

140.1 - $_{\mathrm{Gm} 12}-2975576$

143.2 Gm12_2331255

$146.4-G m 12 \_1883937$

161.0] $\mathrm{Gm} 12849243$

161.3. Gm12 78066

162.3 $=1 \mathrm{Gm} 12$

102.6 Gm12_277889 Gm12 352935

163.3 $4 \mathrm{Gm} 12$ _632910 Gm12_553862

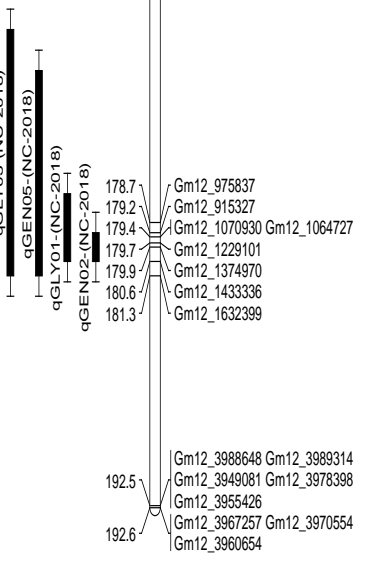

Figure 3. Cont. 

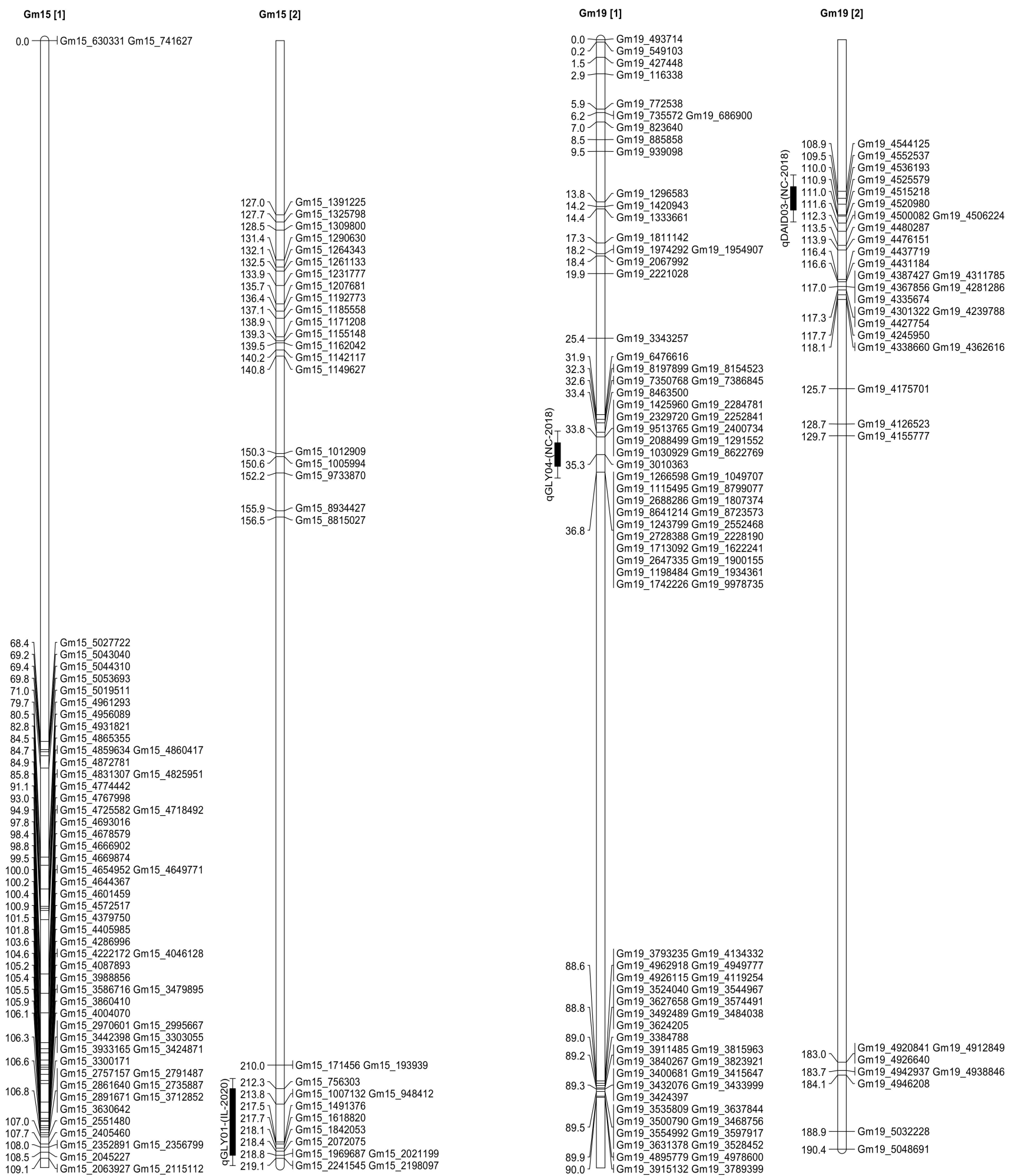

Figure 3. Cont. 

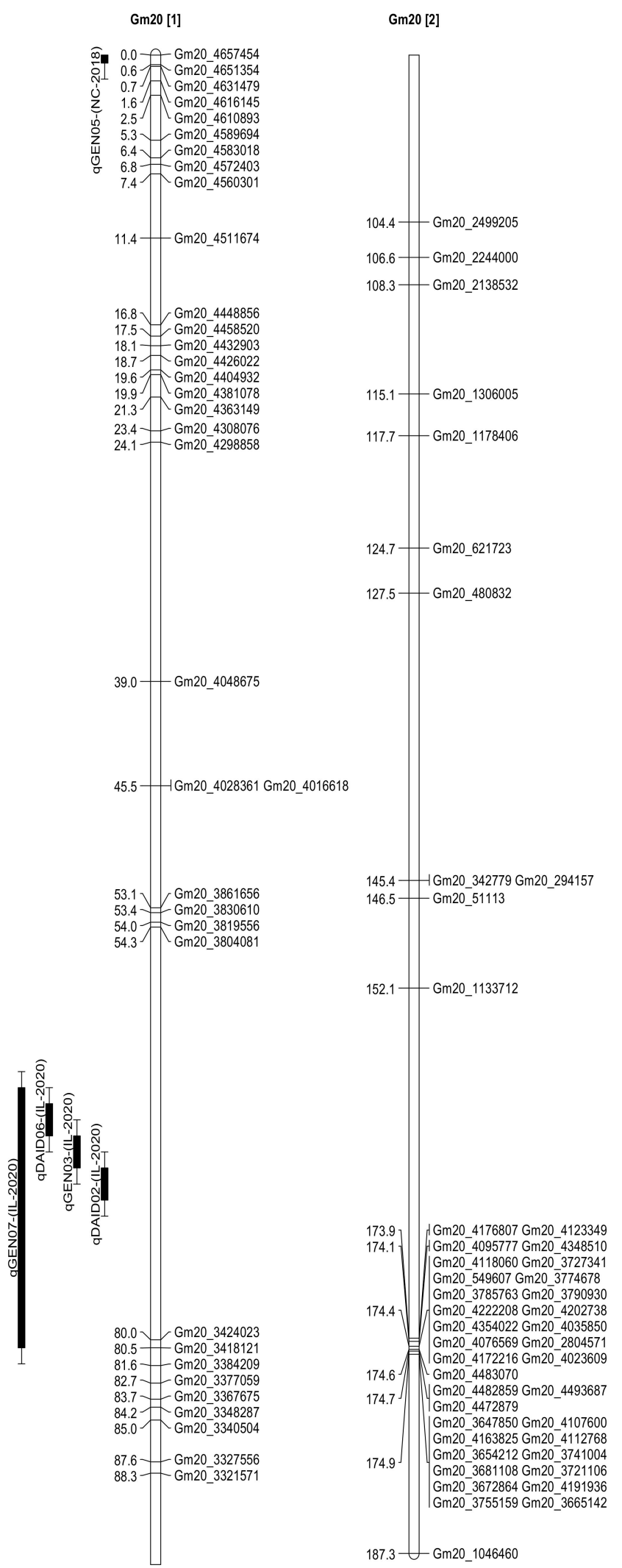

Figure 3. Positions of QTL that control seed genistein (qGEN), daidzein (qDAID), and glycitein (qGLY) contents on Chrs. 2, $4,5,6,10,12,15,19$, and 20. QTL names are followed by a number, location, and year in which they are identified. For example, qGEN01-(NC-2018). The full SNP-based genetic linkage map of Forrest by Williams 82 recombinant inbred line (RIL) population $(n=306)$ of soybean is shown in Figure S2. 
In Carbondale, IL (IL-2020), one QTL that controls seed daidzein content (qDAID03) has been identified and mapped on Chr. 2 and one QTL that controls seed genistein content ( $q$ GEN04) has been identified and mapped on Chr. 4 (Table 4, Figure 3 and Figure S1). One QTL that controls seed daidzein content (qDAID04) and two QTL that control seed genistein content ( $q$ GEN01 and qGEN05) have been identified and mapped on Chr. 10. Two QTL that control each of seed daidzein (qDAID01 and qDAID05) and seed genistein contents ( $q$ GEN02 and $q$ GEN06) have been identified and mapped on Chr. 12 (Table 4, Figure 3 and Figure S1). One QTL that controls seed glycitein (qGLY01) has been identified and mapped on Chr. 15 (Table 4, Figure 3 and Figure S1). Two QTL that control each of seed daidzein ( $q D A I D 02$ and $q D A I D 06)$ and genistein contents ( $q$ GEN03 and $q$ GEN07) have been identified and mapped on Chr. 20 (Table 4, Figure 3 and Figure S1).

In Spring Lake, NC (NC-2020), one QTL that controls each of seed daidzein (qDAID01), genistein ( $q$ GENO3), and glycitein contents ( $q$ GLY02) have been identified and mapped on Chr. 5 (Table 4, Figure 3 and Figure S1). Two QTL that control seed genistein content ( $q$ GEN01 and qGEN04) and one QTL that controls seed daidzein content (qDAID02) have been identified and mapped on Chr. 6 (Table 4, Figure 3 and Figure S1). Two QTL that control each of seed genistein ( $q$ GENO2 and qGEN05) and glycitein contents ( $q$ GLY01 and qGLYO3) have been identified and mapped on Chr. 12 (Table 4, Figure 3 and Figure S1). One QTL that controls each of seed daidzein ( $q D A I D 03$ ) and glycitein contents ( $q$ GLY04) have been identified and mapped on Chr. 19 (Table 4, Figure 3 and Figure S1). One QTL that controls seed genistein content ( $q$ GEN06) has been identified and mapped on Chr. 20 (Table 4, Figure 3 and Figure S1). No QTL that controls total seed isoflavone contents has been identified in both years and locations.

No previous studies identified QTL that control seed isoflavone contents in the QTL region identified on Chr. 2 ( $q$ DAID03-(IL-2020), 23-25 cM), indicating that this is a novel QTL region; however, other studies identified QTL that control seed calcium content, plant height, and few other traits $[40,41]$. Likewise, no other studies identified QTL that control seed isoflavone contents in the QTL region identified on Chr. 4 (qGEN04-(IL-2020), 189.3-191.3 cM) which indicates that it is also a novel QTL region. The length of Chr. 4 in the soybean consensus map is only $136 \mathrm{cM}[29,31]$. Additionally, no other studies identified QTL that control seed isoflavone contents in the QTL region identified on Chr. 5 ( $q$ DAID01(NC-2018), qGEN03-(NC-2018), and qGLY02-(NC-2018), 152.4-166.4 cM) which indicates the discovery of a novel QTL region. The length of Chr. 5 in the soybean consensus map is only $104 \mathrm{cM}[29,31]$. Interestingly, within the same QTL region that controls seed genistein and daidzein contents on Chr. 6 (q-GEN01-(NC-2018), qGEN04-(NC-2018), and qDAID02-(NC-2018), other studies identified QTL that control seed genistein, daidzein, glycitein, and total isoflavone contents (see a summary in [30]) which is coherent with our results making it an important genomic region to further investigate for candidate genes. Other studies identified QTL that control seed protein, oil, $\gamma$-tocopherol, and amino acids contents, and few other traits [29,31]. Interestingly, within the same QTL region that controls seed genistein and daidzein contents on Chr. 10 (q-GEN01-(IL-2020), qGEN05-(IL2020), and qDAID04-(IL-2020), 128.8-132.9 cM), another study identified QTL that control seed isoflavone content [41,42] which is consistent with our data making it an important genomic region for gene discovery. In fact, two candidate genes have been previously identified in this region [42,43]. Two QTL regions have been identified on Chr. 12. The first region containing QTL that control seed genistein and daidzein contents ( $q$ GEN02(IL-2020), qGEN06-(IL-2020), and qDAID05-(IL-2020), 51.2-71.9 cM). Interestingly, other studies identified QTL that control seed daidzein, genistein, glycitein, and total isoflavone contents in the same QTL region (see a summary in [30]) which makes it an important genomic region for discovering novel candidate genes. The second region contained QTL that control seed genistein, and glycitein contents (qGEN02-(NC-2018), qGEN05-(NC-2018), qGLY01-(NC-2018), and qGLY03-(NC-2018), 169.3-181.3 cM). No other studies identified QTL that control seed isoflavone contents in this second QTL region which indicates that it is also a novel QTL region. The length of Chr. 12 in the soybean consensus map is only 
$125 \mathrm{cM}$ and the second QTL region identified here falls outside of its current limit [29,31]. No previous studies identified QTL that control seed isoflavone contents in the QTL region identified on Chr. 15 (qGLY01-(IL-2020), 212.3-218.8 cM) which indicates that it is a novel QTL region. The length of Chr. 15 in the soybean consensus map is only $85 \mathrm{cM}$ and the QTL region identified here falls outside of its current limit [29,31]. Two QTL regions have been identified on Chr. 19. The first region contains QTL that control seed glycitein content (qGLY04-(NC-2018), 34.3-36.3 cM). Interestingly, other studies identified QTL that control seed genistein, daidzein, and isoflavone content within the same QTL region (see a summary in [30]). Previous studies identified also QTL for seed protein content (see a summary in [44]). The second region contained QTL that control (qDAID03-(NC-2018), $108.5-110.5 \mathrm{cM})$. No other studies identified QTL that control seed isoflavone contents in this QTL region, making it a novel QTL region. Two QTL regions have been identified on Chr. 20. Within the first region containing QTL that control seed glycitein content (qGEN05-(NC-2018), 0-2 cM), other studies identified QTL that control seed daidzein ( $q D 20)$, genistein $(q G 20)$, malonyldaidzein $(q M D 20)$, malonylgenistein ( $q M G 20)$, and total isoflavone content (qTIF20) [41,44] which makes it an important region to investigate further for candidate genes. In addition, other studies identified QTL for seed calcium [30,44] and sucrose contents within this QTL region as well (see a summary in [44]). Within the second region containing QTL that control seed daidzein and genistein contents ( $q D A I D 02-$ (IL-2020), qDAID06-(IL-2020), qGEN03-(IL-2020), and qGEN07-(IL-2020), 64.3-80.5 cM), other studies identified QTL that control seed genistein content (qGEN20, [17]) and seed daidzein and glycitein contents ( $q$ GC I proI_1 and qDZ I proI_2, [39,41] which makes it another important region to investigate further for candidate genes. In addition, other studies identified QTL that control seed phytate, stearic acid, calcium, alpha-tocopherol, and few amino acids [44].

\subsection{Seed Isoflavone Candidate Genes}

A total of 130 candidate genes involved in soybean isoflavone biosynthetic pathway have been identified in all 20 soybean Chrs. (Table S1); however, 16 candidate genes have been identified within or close to the seed isoflavone QTL identified in this study on Chrs. 2, 6, 10, 12, 15, 19, and 20 (Figure 4, Table 5).

Among them, the 4'-methoxyisoflavone 2-hydroxylase gene (Glyma.02G067900) and the chalcone synthase gene (Glyma.02G130400) are located at 3.7 and $11.11 \mathrm{cM}$, respectively, from qDAID03-(NC-2018) on Chr. 2 (Figure 4, Table 5 and Table S1). Glyma.06G128200 is a flavonol synthase gene located at $5.52 \mathrm{cM}$ from qGEN01-(NC-2018), qDAID02-(NC2018), and qGEN04-(NC-2018) on Chr. 6. The flavonol 3-O-methyltransferase genes (Glyma.06G137100 and Glyma.06G137300) as well as the chalcone-flavonone isomerase gene (Glyma.06G143000) are located at $6 \mathrm{cM}$ from qGEN01-(NC-2018) on Chr. 6 (Figure 4, Table 5 and Table S1). Glyma.10G058200 is a phenylalanine ammonia-lyase gene that is located 0.6 cM from qGEN01-(IL-2020), qDAID04-(IL-2020) and qGEN05-(IL-2020) on Chr. 10 (Figure 4, Table 4 and Table S1). Glyma.12G067000 and Glyma.12G067100 are located within qDAID01-(IL-2020), qGEN02-(IL-2020) and qDAID05-(IL-2020) on Chr. 12 and near to (<4 cM) qGEN02-(NC-2018), qGLY01-(NC-2018), qGEN05-(NC-2018), qGEN06-(IL-2020) and qGLY03-(NC-2018) on Chr. 12 (Figure 4, Table 5 and Table S1). Both genes are $\mathrm{Cy}-$ tochrome P450 CYP2 subfamily genes; Glyma.12G067000 was classified as an isoflavone synthase II gene and Glyma.12G067100 as its duplicate with 95\% identical nucleotide positions in the protein coding sequence ([42] Fliegmann et al., 2010). The trans-feruloyl-CoA synthase gene Glyma.15G001700 is located at 0.56 cM from qGLY01-(IL-2020) on Chr. 15. The Isoflavone 3'-hydroxylase gene Glyma.15G156300 and the Isoflavone 2'-hydroxylase gene Glyma.15G156100 are located at about $11 \mathrm{cM}$ from qGLY01-(IL-2020) on Chr. 15 (Figure 4, Table 5 and Table S1). Glyma.20G027800 is an isoflavone reductase gene that is located within qDAID06-(IL-2020) and at $0.62 \mathrm{cM}$ from qDAID02-(IL-2020), qGEN03-(IL-2020), and qGEN06-(NC-2018) and $2.44 \mathrm{cM}$ from qGEN07-(IL-2020) on Chr. 20 (Figure 4, Table 5 and Table S1). Three genes Glyma.19G030500, Glyma.19G030700, and Glyma.19G030800 
encoding for an isoflavone 7-O-glucoside-6"-O-malonyltransferase gene family are located at less than $1 \mathrm{cM}$ from qDAID03-(NC-2018) and qGLY04-(NC-2018) on Chr. 19 (Figure 4, Table 5 and Table S1). Interestingly, Wu et al. (2020) identified seven candidate genes including the mitogen-activated protein kinase (MPK) gene (Glyma.08G309500) within the seed isoflavone QTL identified on Chr. 8 [29,31]. A summary of seed isoflavone QTL and corresponding candidate genes for over two decades of research (1999-2020) can be found in Kassem [30]. Recently, Yang et al. (2021) [32] identified four candidate genes including GSTT1a (Glyma.05G206900), GSTT1b (Glyma.05G207000), and the transcription factor (TF) GL3 (Glyma.05G208300) on Chr. 5, and GSTL3 (Glyma.13G135600) on Chr. 13 [32].

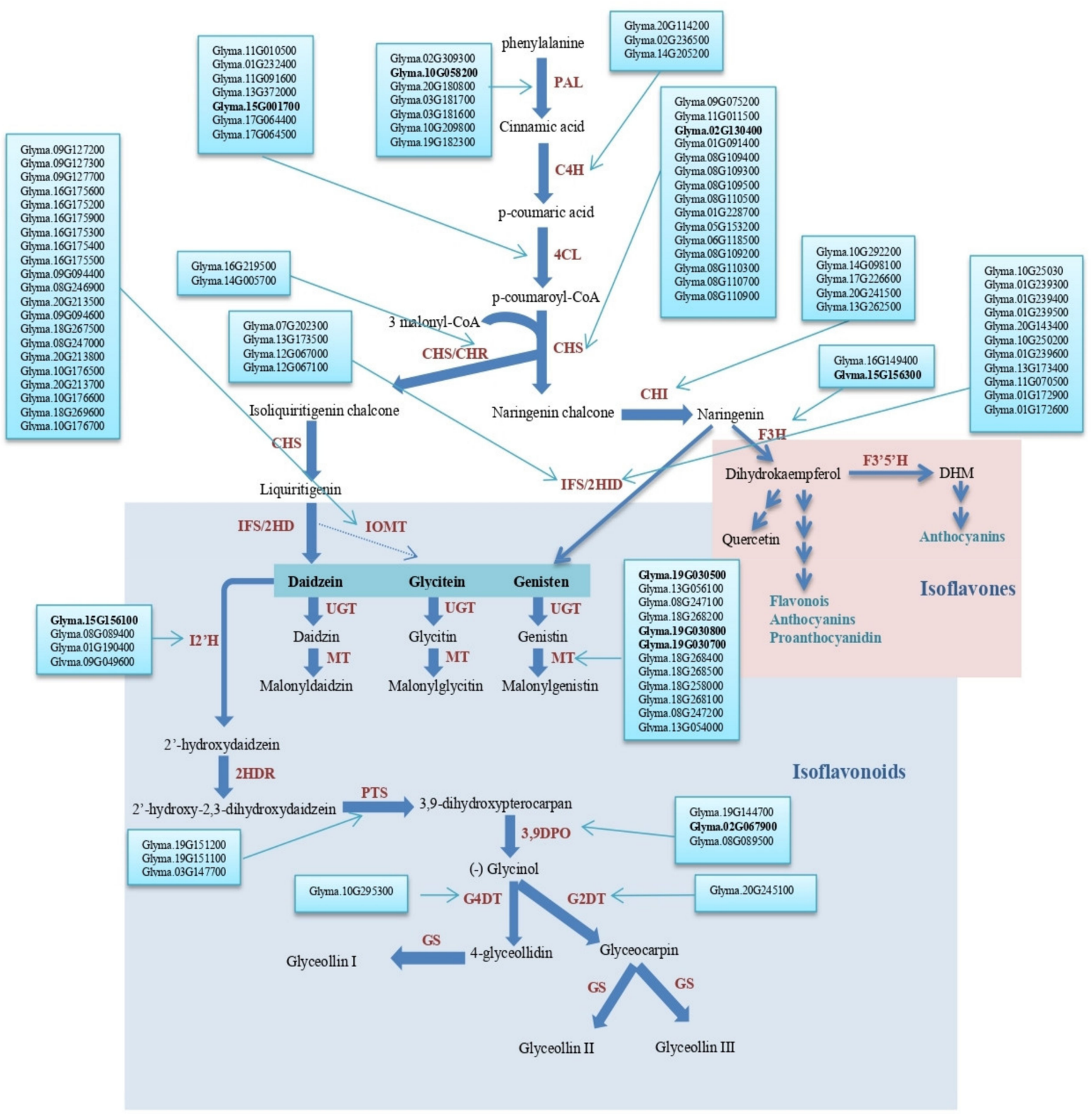

Figure 4. Seed isoflavone metabolic pathway in soybean with identified candidate genes (Vadivel et al., 2010). PAL, phenylalanine ammonia lyase; $\mathrm{C} 4 \mathrm{H}$, cinnamate 4-hydroxylate; 4CL, 4-coumarate-CoA ligase; CHS, chalcone synthase; $\mathrm{CHR}$, chalcone reductase; $\mathrm{CHI}$, chalcone isomerase; IFS, 2-hydroxyisoflavanone synthase; 2HID, 2-hydroxyisoflavanone dehydratase; IOMT, isoflavone O-methyltransferase; UGT, uridine diphosphate glycosyltransferase; MT, malonyltransferase; I2' H, Isoflavone 2'-hydroxylase; $2 \mathrm{HDR}, 2^{\prime}$-hydroxydaidzein reductase; F3H, flavanone-3-hydroxylase; F3' $5^{\prime} \mathrm{H}$, flavonoid 3'5'hydroxylase; DHM, dihydromyricetin; PTS, pterocarpan synthase; 3,9 DPO, 3,9-dihydroxypterocarpan 6a-monooxygenase; G4DT, glycinol 4-dimethylallyltransferase; G2DT, glycinol 2-dimethylallyltransferase; GS, glyceollin synthase. 
Table 5. Isoflavone candidate genes located within or close to the isoflavone QTL identified in the FxW82 RIL population two environments over two years (A. Spring Lake, NC (2018) and B. Carbondale, IL (2020)).

(A).

\begin{tabular}{|c|c|c|c|c|c|c|c|}
\hline Environment & Trait & QTL & Chr. & Gene & Start & End & Distance (cM) \\
\hline \multicolumn{8}{|c|}{2018 CIM QTL with LOD Scores > 2.5} \\
\hline \multirow{4}{*}{ Spring Lake, NC } & \multirow{4}{*}{ Genistein } & \multirow{4}{*}{ qGEN01 } & \multirow{4}{*}{6} & Glyma.06G128200 & $10,543,911$ & $10,545,747$ & $5.52 \mathrm{cM}$ \\
\hline & & & & Glyma.06G137100 & $11,225,188$ & $11,228,664$ & $6.21 \mathrm{cM}$ \\
\hline & & & & Glyma.06G137300 & $11,237,072$ & $11,239,469$ & $6.22 \mathrm{cM}$ \\
\hline & & & & Glyma.06G143000 & $11,642,031$ & $11,644,022$ & $6.62 \mathrm{cM}$ \\
\hline \multirow{2}{*}{ Spring Lake, NC } & \multirow{2}{*}{ Genistein } & \multirow{2}{*}{ qGEN02 } & \multirow{2}{*}{12} & Glyma.12G067000 & $4,909,073$ & $4,911,905$ & $3.47 \mathrm{cM}$ \\
\hline & & & & Glyma.12G067100 & $4,919,960$ & $4,922,998$ & $3.48 \mathrm{cM}$ \\
\hline \multirow{2}{*}{ Spring Lake, NC } & \multirow{2}{*}{ Glycitein } & \multirow{2}{*}{ qGLY01 } & \multirow{2}{*}{12} & Glyma.12G067000 & $4,909,073$ & $4,911,905$ & $3.47 \mathrm{cM}$ \\
\hline & & & & Glyma.12G067100 & $4,919,960$ & $4,922,998$ & $3.48 \mathrm{cM}$ \\
\hline \multicolumn{8}{|c|}{2018 CIM QTL with LOD Scores $2.0<$ LOD $<2.5$} \\
\hline Spring Lake, NC & Daidzein & qDAID01 & 5 & - & - & - & - \\
\hline \multirow[t]{2}{*}{ Spring Lake, NC } & Daidzein & $q D A I D 02$ & 6 & Glyma.06G128200 & $10,543,911$ & $10,545,747$ & $5.52 \mathrm{cM}$ \\
\hline & & & & Glyma.19G030500 & $3,779,017$ & $3,781,453$ & $0.77 \mathrm{cM}$ \\
\hline \multirow[t]{2}{*}{ Spring Lake, NC } & Daidzein & qDAID03 & 19 & Glyma.19G030700 & $3,794,404$ & $3,796,426$ & $0.75 \mathrm{cM}$ \\
\hline & & & & Glyma.19G030800 & $3,799,941$ & $3,801,335$ & $0.75 \mathrm{cM}$ \\
\hline Spring Lake, NC & Genistein & qGEN03 & 5 & - & & & \\
\hline Spring Lake, NC & Genistein & qGEN04 & 6 & Glyma.06G128200 & $10,543,911$ & $10,545,747$ & $5.52 \mathrm{cM}$ \\
\hline \multirow{2}{*}{ Spring Lake, NC } & \multirow{2}{*}{ Genistein } & \multirow{2}{*}{ qGEN05 } & \multirow{2}{*}{12} & Glyma.12G067000 & $4,909,073$ & $4,911,905$ & $3.27 \mathrm{cM}$ \\
\hline & & & & Glyma.12G067100 & $4,919,960$ & $4,922,998$ & $3.28 \mathrm{Cm}$ \\
\hline Spring Lake, NC & Genistein & qGEN06 & 20 & Glyma.20G027800 & $3,179,955$ & $3,183,453$ & $1.47 \mathrm{cM}$ \\
\hline Spring Lake, NC & Glycitein & qGLY02 & 5 & - & - & - & - \\
\hline \multirow{2}{*}{ Spring Lake, NC } & \multirow{2}{*}{ Glycitein } & \multirow{2}{*}{ qGLY03 } & \multirow[b]{2}{*}{12} & Glyma.12G067000 & $4,909,073$ & $4,911,905$ & $3.27 \mathrm{cM}$ \\
\hline & & & & Glyma.12G067100 & $4,919,960$ & $4,922,998$ & $3.28 \mathrm{Cm}$ \\
\hline \multirow{3}{*}{ Spring Lake, NC } & \multirow{3}{*}{ Glycitein } & \multirow{3}{*}{ qGLY04 } & \multirow{3}{*}{19} & Glyma.19G030500 & $3,779,017$ & $3,781,453$ & $0.76 \mathrm{cM}$ \\
\hline & & & & Glyma.19G030700 & $3,794,404$ & $3,796,426$ & $0.78 \mathrm{cM}$ \\
\hline & & & & Glyma.19G030800 & $3,799,941$ & $3,801,335$ & $0.78 \mathrm{cM}$ \\
\hline
\end{tabular}

(B).

\begin{tabular}{|c|c|c|c|c|c|c|c|}
\hline Environment & Trait & QTL & Chr. & Gene & Start & End & Distance (cM) \\
\hline \multicolumn{8}{|c|}{2020 CIM QTL with LOD Scores > 2.5} \\
\hline \multirow{2}{*}{ Carbondale, IL } & \multirow[b]{2}{*}{ Daidzein } & \multirow{2}{*}{$q D A I D 01$} & \multirow{2}{*}{12} & Glyma.12G067000 & $4,909,073$ & $4,911,905$ & - \\
\hline & & & & Glyma.12G067100 & $4,919,960$ & $4,922,998$ & - \\
\hline Carbondale, IL & Daidzein & qDAID02 & 20 & Glyma.20G027800 & $3,179,955$ & $3,183,453$ & $0.62 \mathrm{cM}$ \\
\hline Carbondale, IL & Genistein & qGEN01 & 10 & Glyma.10G058200 & $5,328,963$ & $5,333,501$ & $0.6 \mathrm{cM}$ \\
\hline \multirow{2}{*}{ Carbondale, IL } & \multirow{2}{*}{ Genistein } & \multirow{2}{*}{ qGEN02 } & \multirow{2}{*}{12} & Glyma.12G067000 & $4,909,073$ & $4,911,905$ & - \\
\hline & & & & Glyma.12G067100 & $4,919,960$ & $4,922,998$ & - \\
\hline Carbondale, IL & Genistein & qGEN03 & 20 & Glyma.20G027800 & $3,179,955$ & $3,183,453$ & $2.44 \mathrm{cM}$ \\
\hline \multicolumn{8}{|c|}{2020 CIM QTL with LOD Scores $2.0<$ LOD $<2.5$} \\
\hline \multirow{2}{*}{ Carbondale, IL } & \multirow[b]{2}{*}{ Daidzein } & \multirow{2}{*}{$q D A I D 03$} & \multirow[b]{2}{*}{2} & Glyma.02G067900 & $5,986,285$ & $5,987,684$ & $3.70 \mathrm{cM}$ \\
\hline & & & & Glyma.02G130400 & $13,399,253$ & $13,401,493$ & $11.11 \mathrm{cM}$ \\
\hline Carbondale, IL & Daidzein & qDAID04 & 10 & Glyma.10G058200 & $5,328,963$ & $5,333,501$ & $0.6 \mathrm{cM}$ \\
\hline \multirow{2}{*}{ Carbondale, IL } & \multirow{2}{*}{ Daidzein } & \multirow{2}{*}{ qDAID05 } & \multirow{2}{*}{12} & Glyma.12G067000 & $4,909,073$ & $4,911,905$ & - \\
\hline & & & & Glyma.12G067100 & $4,919,960$ & $4,922,998$ & - \\
\hline Carbondale, IL & Daidzein & qDAID06 & 20 & Glyma.20G027800 & $3,179,955$ & $3,183,453$ & - \\
\hline Carbondale, IL & Genistein & qGEN04 & 4 & - & - & - & - \\
\hline Carbondale, IL & Genistein & qGEN05 & 10 & Glyma.10G058200 & $5,328,963$ & $5,333,501$ & $0.6 \mathrm{cM}$ \\
\hline \multirow{2}{*}{ Carbondale, IL } & \multirow{2}{*}{ Genistein } & \multirow{2}{*}{ qGEN06 } & \multirow[b]{2}{*}{12} & Glyma.12G067000 & $4,909,073$ & $4,911,905$ & $3.48 \mathrm{cM}$ \\
\hline & & & & Glyma.12G067100 & $4,919,960$ & $4,922,998$ & $3.49 \mathrm{cM}$ \\
\hline \multirow[t]{2}{*}{ Carbondale, IL } & \multirow[t]{2}{*}{ Genistein } & \multirow[t]{2}{*}{ qGEN07 } & \multirow[t]{2}{*}{20} & Glyma.20G027800 & $3,179,955$ & $3,183,453$ & $2.44 \mathrm{cM}$ \\
\hline & & & & Glyma.15G001700 & 190,985 & 194,451 & $0.56 \mathrm{cM}$ \\
\hline \multirow[t]{2}{*}{ Carbondale, IL } & \multirow[t]{2}{*}{ Glycitein } & \multirow[t]{2}{*}{ qGLY01 } & \multirow[t]{2}{*}{15} & Glyma.15G156300 & $13,098,492$ & $13,100,036$ & $11.02 \mathrm{cM}$ \\
\hline & & & & Glyma.15G156100 & $13,076,997$ & $13,079,333$ & $11 \mathrm{cM}$ \\
\hline
\end{tabular}




\subsection{Expression Analysis}

To gain insight into the role of isoflavone genes in soybean seeds, RNA-Seq analysis was conducted to check the expression levels of the 16 candidate genes that are located within or near the isoflavone QTL identified in FxW82 RIL population. Expression analysis of these genes showed that four genes, Glyma.10G058200, Glyma.06G143000, Glyma.06G137100, and Glyma.06G137300, are highly expressed in seeds of both Forrest and Williams 82 cultivars. Whereas, Glyma.19G030800 is highly expressed in Williams 82 and have a low expression in Forrest cv.; the rest of the 16 genes showed lower expressions; whereas Glyma.02G067900 and Glyma.15G156300 were not expressed neither in Forrest nor in Williams 82 cultivars (Figure 5).
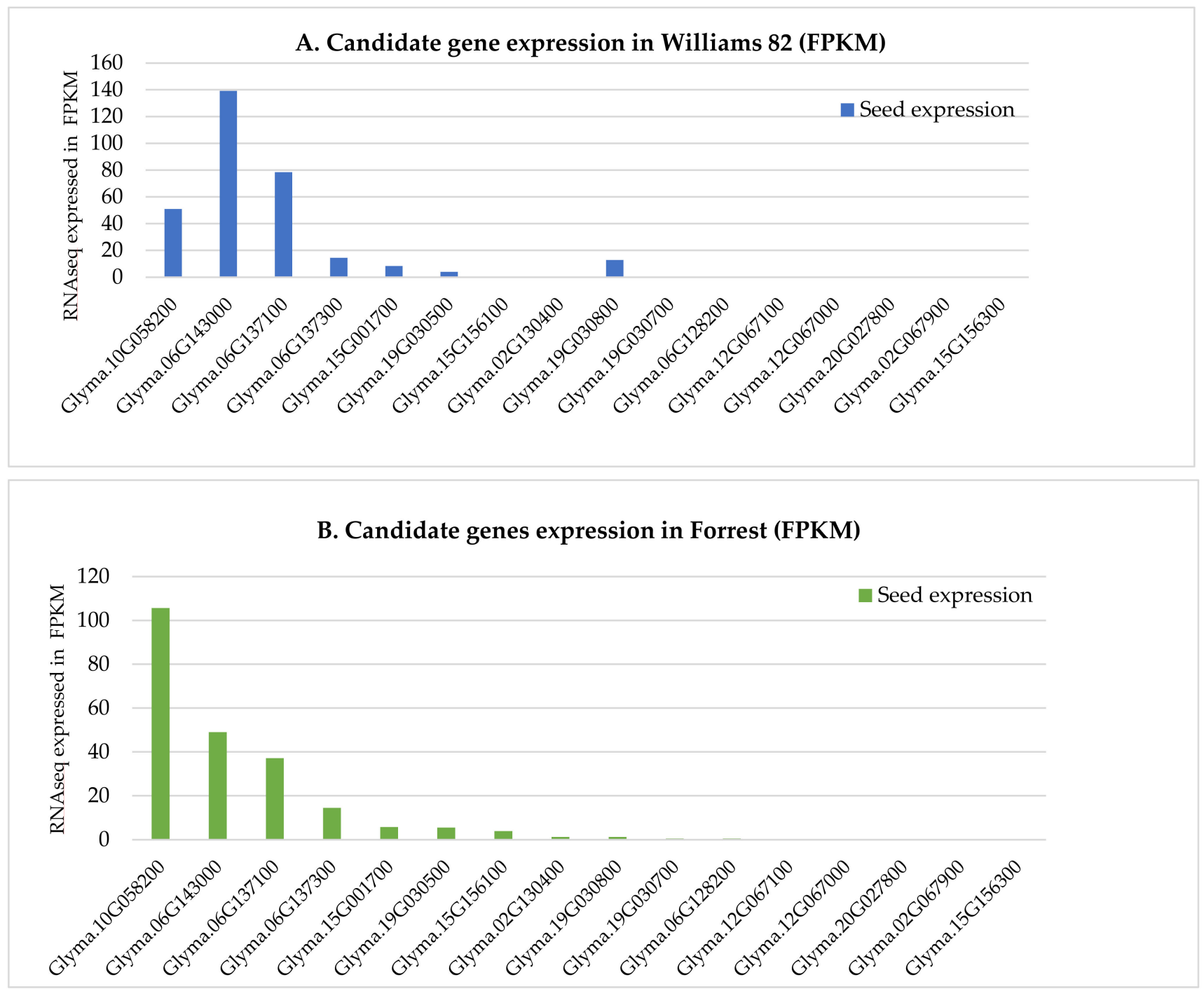

Figure 5. Expression pattern of isoflavone genes in soybean seeds. (A) Expression of the 16 isoflavone genes located within isoflavone QTL in Williams 82 (FPKM) were retrieved from publicly available RNA-seq data from Phytozome database [45], in addition to (B) the RNAseq data from the cultivar 'Forrest' (FPKM).

Surprisingly, Glyma.10G058200 expression in Forrest cv. is higher than its expression in Williams 82. This could explain the presence of RILs from the FxWI cross that showed higher daidzein, glycitein or genistein content than the parent Williams 82 (parent with the high isoflavones content), these lines inherited most likely the beneficial alleles from both parents Forrest and Williams 82. 


\subsection{Conclusions}

In conclusion, we constructed the FxW82 dense SNP-based genetic linkage map (2075 SNPs and $4029.9 \mathrm{cM}$ covered) and identified 27 QTL that control soybean seed isoflavone contents and 16 candidate genes involved in soybean isoflavone biosynthetic pathways among which four candidate genes are highly expressed in seeds of both Forrest and Williams 82, in addition to Glyma.19G030800 that has a higher expression profile in Williams 82 compared to its expression in Forrest cv. (Figure 5).

A comparison of the Forrest and Williams 82 sequences of these four genes has shown that two of these genes have SNPs between Forrest and Williams 82 sequences, Glyma.10G058200 and Glyma.06G143000. Glyma.10G058200 has ten SNPs, one SNP is upstream $5^{\prime}$ UTR, four SNPs are located at the intron, two SNPs are at the exon 1, one of them caused a missense mutation (A127G) and the other one caused a silent mutation (A32A). The last three are in the 3' UTR downstream region. For Glyma.06G143000, there is only one SNP located in the 5`UTR upstream region (Figure 6). These SNPs could potentially play a role in the difference of isoflavones content between Forrest and Williams 82 cultivars. Moreover, Glyma.10G058200 and Glyma.06G143000 are highly expressed in the seed tissue of both Forrest and Williams 82 (Figure 5). Glyma.10G058200 is associated with qGEN01-(IL-2020) QTL, qDAID04-(IL-2020) QTL and qGEN05-(IL-2020) QTL. Additionally, Glyma.06G143000 is associated with qGEN01-(NC-2018) QTL. The two genes could be useful for breeding for increased isoflavones content in soybeans.

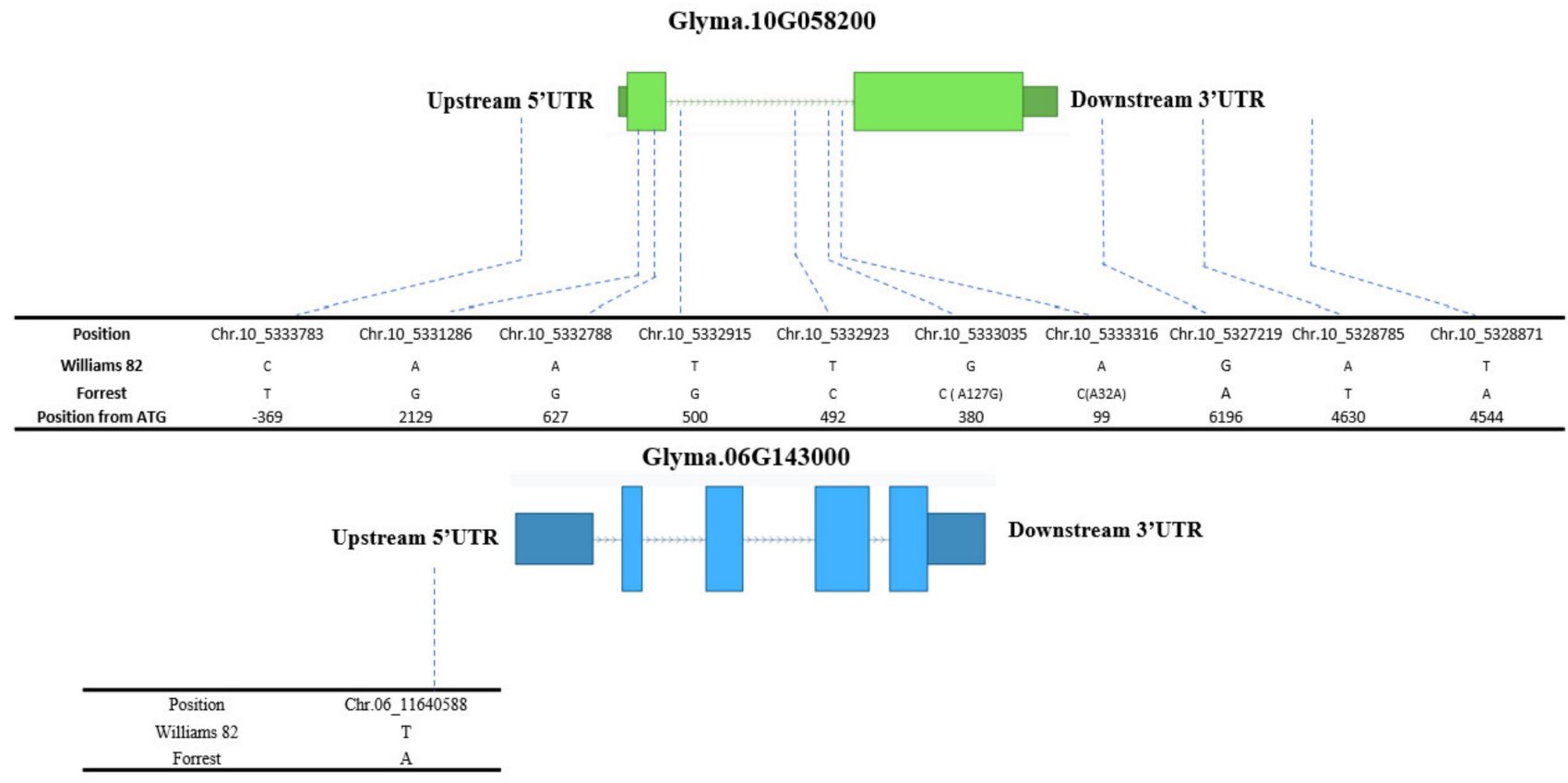

Figure 6. Positions of SNPs between Forrest and Williams 82 cultivars in Glyma.10G058200 and Glyma.06G143000 genes.

\section{Materials and Methods}

\subsection{Plant Material and Growth Conditions}

In this study, we used 'Forrest' $\times$ 'Williams 82 ' RIL population $(n=306)$. The cultivar 'Forrest' was derived from the cross of 'Dyer' and 'Bragg' developed by USDA [46]. The cultivar 'Williams 82' was derived from the cross of 'Williams' and 'Kingwa' [47]. The genomes of soybean cultivars including Forrest and Williams 82 genomes are duplicated polyploid genomes with highly conserved gene-rich regions [48]. Originally, the 'Forrest' $\times$ 'Williams 82' RIL population was developed with more than 1000 RILs [49]. The genetic map used in this study was based on 306 RILs and 2075 SNP markers; however, QTL data analysis in Spring Lake, NC-2018 was based on 190 RILs. 
The RIL population was evaluated in a farm in Spring Lake, NC $\left(35.17^{\circ} \mathrm{N}, 78.97^{\circ} \mathrm{W}\right)$ in 2018 and in a farm in Carbondale, IL $\left(37^{\circ} \mathrm{N}, 89^{\circ} \mathrm{W}\right)$ in 2020 . Seeds of parents (Forrest and Williams 82) were sown directly in the field in a randomized complete block design $(\mathrm{RCB})$ and $75 \mathrm{~cm}$ row spacing between seeds with three replicates. The plants were watered by drip irrigation and kept in the field until maturity. No pesticide, herbicide, or fertilizer were applied. In September 2018, hurricane Florence hit NC and its winds of 90+ mph damaged the fence in the farm in Spring Lake, NC and the deer damaged about 119 RILs; therefore, QTL data analysis for this location involved 187 RILs $(n=187)$. The plants grown in Carbondale, IL $(n=306)$ were not damaged.

In Spring Lake, NC (2018) during the growing season (May-Sept.), the temperatures ranged from 7.2 to $35^{\circ} \mathrm{C}$, it was partly $(40 \%)$ to mostly cloudy $(80 \%)$, wind speeds ranging from 55 to $90+\mathrm{mph}$ (hurricane Florence), and humidity comfort level ranged from comfortable to miserable [50]. The soil type in this location is mainly sandy (NC Sandhills). In Carbondale, IL (2020), the temperatures ranged from 7.2 to $29.4{ }^{\circ} \mathrm{C}$, it was mostly clear $(25 \%)$ to mostly cloudy $(80 \%)$, wind speeds ranging from 30 to $38 \mathrm{mph}$, and humidity comfort level ranged from comfortable to miserable (weatherspark.com). The field was treated first using Firestorm (contains Paraquat dichloride) to control annual grass and broad-leaved weeds. As pre-emergent herbicide, Dual II Magnum Herbicide with longlasting control of most annual grasses and small-seeded broadleaf weeds was used to eliminate early-season weed competition. As post-emergent herbicide, Round Up Pro Concentrate (50.2\% Glyphosate) was used/sprayed between the rows to control emerging weed. Weed grown inside the plastic mulch very close to the soybeans were removed manually. The soil type in this location is mainly silty clay loam (Southern IL).

\subsection{Isoflavone Quantification}

Mature seeds of parents Forrest and Williams 82, and the 190 RILs were analyzed for the concentrations aglycones daidzein, genistein, and glycitein. Approximately $25 \mathrm{~g}$ of mature seeds from each plot were ground using a Laboratory Mill 3600 (Perten, Springfield, IL, USA). Concentrations of daidzein, genistein, and glycitein were analyzed using a nearinfrared reflectance (NIR) diode array feed analyzer (Perten, Spring Field, IL, USA). The calibration equation has been updated every 6 months to 1 year and developed using the Thermo Galactic Grams PLS IQ software developed by Perten Company (Perten, Springfield, IL, USA). Thermo Galactic Grams PLS IQ from Perten (Perten) was used to develop the calibration equations, which was initially developed by the University of Minnesota. Descriptions of quantifying daidzein, genistein, glycitein and total isoflavone contents was reported by others (Akond et al., 2015 [22]; Bellaloui et al., 2012 [51]; Wang et al., 2019 [38]). The calibration equation development and updating for isoflavones was based on standard laboratory analytical methods (AOAC 2002) using High Performance Liquid Chromatography (HPLC) and use of adequate number of samples, providing sufficiently accurate estimations of isoflavones concentrations. The produced calibration equation was characterized by high correlation, indicating the accuracy of the method. The concentrations were calculated on a seed dry matter basis.

\subsection{DNA Isolation, SNP Genotyping, and Genetic Map Construction}

Genomic DNA of the RIL population and the parents were extracted using a standard cetyltrimethyl ammonium bromide (CTAB) method with minor modifications as previously described [52]. DNA concentration was quantified with a spectrophotometer (NanoDrop Technologies Inc., Centreville, DE, USA) and then normalized at $50 \mathrm{ng} / \mu \mathrm{L}$ for genotyping. SNP genotyping was performed in the Soybean Genomics and Improvement Laboratory, USDA-ARS, Beltsville, MD, USA, using the Illumina Infinium SoySNP6K BeadChips (Illumina, Inc. San Diego, CA, USA) as previously described [53]. Subsequently, SNP alleles were called using GenomeStudio Genotyping Module 2.0 (Illumina, Inc. San Diego, CA, USA). 
JoinMap 4.0 [54] was used to construct the genetic linkage map with a LOD score threshold of 3.0 and a maximum genetic distance of $50 \mathrm{cM}$ to group markers. The linkage groups were assigned to corresponding soybean chromosomes as described in SoyBase $[29,31]$.

\subsection{Isoflavone QTL Detection and Statistical Analysis}

The broad sense (mean based) heritability analysis from two-way ANOVA was conducted using the following equation: $\mathrm{h}^{2}=\sigma_{\mathrm{G}}{ }^{2} /\left[\sigma_{\mathrm{G}}{ }^{2}+\left(\sigma_{\mathrm{GE}}{ }^{2} / \mathrm{e}\right)+\left(\sigma_{\mathrm{e}}{ }^{2} / \mathrm{re}\right)\right]$ where $\sigma_{\mathrm{G}}{ }^{2}$ (variance of genetic factor), $\sigma_{\mathrm{GE}}{ }^{2}$ (variance of genotype-environment interactions), and $\sigma_{\mathrm{e}}{ }^{2}$ (variance of random effect) were calculated with e (number of environment) and $\mathrm{r}$ (number of replicates) normalization [55]. R [56] was employed in the statistical analysis including agronomic traits, histogram of trait distribution, two-way ANOVA, and broad sense heritability using its native packages. The significant level of the assessed traits was showed using R package car (type II Wald chi-square tests) [56].

Both interval mapping (IM) and composite interval mapping (CIM) methods of WinQTL Cartographer 2.5 [39] were used to identify QTL for seed genistein, daidzein, glycitein, and total isoflavone contents in this RIL population. The default parameters of WinQTL Cartographer were chosen (Model 6, $1 \mathrm{cM}$ step size, $10 \mathrm{cM}$ window size, 5 control markers, and 1,000 permutations threshold) [39]. Chromosomes were drawn using MapChart 2.2 [57].

\subsection{Isoflavone Candidate Genes Identification}

The Glyma numbers of the isoflavone genes were obtained by searching the available data at the SoyBase $[29,31]$ and Phytozome database [45]. The name of the isoflavone pathway enzymes (Figure 5) were used as a query in a search of the Glycine max reference genome, version Williams 82 . The obtained isoflavone genes were mapped to the identified isoflavone QTL.

\subsection{Expression Analysis}

The expression analysis of the genes that are located within or near the isoflavone QTL was conducted using the publicly available soybean expression database from Phytozome database [45] to infer expression profiles of isoflavone genes in the soybean reference genome Williams 82. Gene expression was estimated in FPKM (Fragments Per Kilobase of transcript per Million mapped reads).

For the Forrest cv., RNA-seq library was prepared by using four plant soybean tissues including seed, leaf, root, flower and pods as shown earlier [58]. From $100 \mathrm{mg}$ of frozen grounded samples, total RNA was extracted using RNeasy QIAGEN Kit (Qiagen, Hilden, Germany). The DNase I (Invitrogen, Carlsbad, CA, USA) was used to treat the total RNA. Using Illumina NovaSeq 6000, RNA-seq libraries preparation and sequencing were performed at Novogene INC. Multiplexing and sequencing of the four libraries were done in two different lanes generating 20 million raw pair end reads per sample (150 bp). Quality of sequenced reads was assessed using fastqc, version 0.11.9. [59]. The low-quality reads and adapters were removed with trimmomatic, version V0.39, the remaining highquality reads were mapped to the soybean reference genome Wm82.a2.v1 using STAR, version v2.7.9 [60,61]. Uniquely mapped reads were counted using Python package HTseq v0.13.5. [62]. Read count normalization and differential gene expression analysis were conducted using the Deseq2 package v1.30.1 [63] integrated in the OmicsBox platform from BioBam (Valencia, Spain) [58,64].

Supplementary Materials: The following are available online at https:/ /www.mdpi.com/article/10 .3390/plants10102029/s1, Figure S1: Positions of major QTL (LOD > 2.5) that control seed genistein (qGEN), daidzein (qDAID), and glycitein (qGLY) contents identified in the FXW82 RIL population. (A) QTL identified in Spring Lake, NC (2018) and (B) QTL identified in Carbondale, IL (2020); Figure S2. The SNP-based genetic linkage map of Forrest by Williams 82 recombinant inbred line (RIL) population $(n=306)$ of soybean. Chrs. were drawn using MapChart 2.2 (Voorrips, 2002 [57]). Positions of QTL that control seed genistein (qGEN), daidzein (qDAID), and glycitein 
(qGLY) contents are indicated with black bars on Chrs. 2, 4, 5, 6, 10, 12, 15, 19, and 20. QTL names are followed by a number, location, and year in which they are identified. For example, qGEN01-(NC2018); Table S1: Candidate genes involved in soybean isoflavone biosynthetic pathways and their Phytozome annotation. These 130 candidate genes are identified in all $20 \mathrm{Chrs}$.

Author Contributions: Conceptualization, M.A.K. and K.M.; methodology, D.K., J.Y., T.V., M.U., Q.S., F.B., T.R., E.W., N.L., N.B. and A.M.; validation, M.A.K., K.M. and H.T.N.; formal analysis, D.K., J.Y. and A.M.; investigation, K.M., D.K. and A.M.; resources, K.M., N.B., A.M., and H.T.N.; genotyping, Q.S., data curation, D.K., J.Y., M.U. and T.V.; writing-original draft preparation, M.A.K. and D.K.; writing-review and editing, D.K., J.Y., N.B., N.L., H.M., T.V., A.M., M.A.K., K.M. and H.T.N.; visualization, J.Y.; supervision, M.A.K., J.Y., K.M., H.M., H.T.N.; project administration, M.A.K., K.M. and H.T.N. All authors have read and agreed to the published version of the manuscript.

Funding: This research was funded by the U.S. Department of Agriculture, Agricultural Research Service Project 6066-21220-014-00D, SIUC, UM, and FSU. All authors have read and agreed to the published version of the manuscript.

Data Availability Statement: Data supporting reported results are available on request from the corresponding author.

Acknowledgments: Technical support provided by S. Mosley is appreciated. Mention of trade names or commercial products in this publication is solely for the purpose of providing specific information and does not imply recommendation or endorsement by the United States Department of Agriculture (USDA). USDA is an equal opportunity provider and employer.

Conflicts of Interest: The authors declare no conflict of interest.

\section{References}

1. Cavallini, D.C.U.; Bedani, R.; Bomdespacho, L.Q.; Vendramini, R.C.; Rossi, E.A. Effects of probiotic bacteria, isoflavones and simvastatin on lipid profile and atherosclerosis in cholesterol-fed rabbits: A randomized double-blind study. Lipids Health Dis. 2009, 8, 1. [CrossRef]

2. Cederroth, C.R.; Zimmermann, C.; Nef, S. Soy, phytoestrogens and their impact on reproductive health. Mol. Cell. Endocrinol. 2012, 355, 192-200. [CrossRef]

3. Kwon, Y. Effect of soy isoflavones on the growth of human breast tumors: Findings from preclinical studies. Food Sci. Nutr. 2014, 2, 613-622. [CrossRef]

4. Setchell, K.D.R.; Cassidy, A. Dietary Isoflavones: Biological Effects and Relevance to Human Health. J. Nutr. 1999, 129, 758S-767S. [CrossRef]

5. Cederroth, C.R.; Nef Soy, S. phytoestrogens and metabolism: A review. Mol. Cell. Endocrinol. 2009, 304, 30-42. [CrossRef]

6. Jiang, Q.; Payton-Stewart, F.; Elliott, S.; Driver, J.; Rhodes, L.V.; Zhang, Q.; Zheng, S.; Bhatnagar, D.; Boue, S.M.; Collins-Burow, B.M.; et al. Effects of 7-O Substitutions on Estrogenic and Anti-Estrogenic Activities of Daidzein Analogues in MCF-7 Breast Cancer Cells. J. Med. Chem. 2010, 53, 6153-6163. [CrossRef]

7. Hamilton-Reeves, J.M.; Banerjee, S.; Banerjee, S.K.; Holzbeierlein, J.M.; Thrasher, J.B.; Kambhampati, S.; Keighley, J.; Veldhuizen, P.V. Short-Term Soy Isoflavone Intervention Patients with Localized Prostate Cancer: A Randomized, Double-Blind, Placebo-Controlled Trial. PLoS ONE 2013, 8, e68331. [CrossRef] [PubMed]

8. Fritz, H.; Seely, D.; Flower, G.; Skidmore, B.; Fernandes, R.; Vadeboncoeur, S.; Kennedy, D.; Cooley, K.; Wong, R.; Sagar, S.; et al. Soy, Red Clover, and Isoflavones and Breast Cancer: A Systematic Review. PLoS ONE 2013, 8, e81968. [CrossRef]

9. Chen, M.; Rao, Y.; Zheng, Y.; Wei, S.; Li, Y.; Guo, T.; Yin, P. Association between Soy Isoflavone Intake and Breast Cancer Risk for Pre- and Post-Menopausal Women: A Meta-Analysis of Epidemiological Studies. PLoS ONE 2014, 9, e89288. [CrossRef] [PubMed]

10. Wei, J.; Bhatt, S.; Chang, L.M.; Sampson, H.A.; Masilamani, M. Isoflavones, Genistein and Daidzein, Regulate Mucosal Immune Response by Suppressing Dendritic Cell Function. PLoS ONE 2012, 7, e47979. [CrossRef] [PubMed]

11. Sakai, T.; Kogiso, M. Soy isoflavones and immunity. J. Med. Investig. 2008, 55, 167-173. [CrossRef] [PubMed]

12. Subramanian, S.; Stacey, G.; Yu, O. Distinct, crucial roles of flavonoids during legume nodulation. Trends Plant Sci. 2007, 12, 282-285. [CrossRef]

13. Zhang, Y.; Wang, G.-J.; Song, T.T.; Murphy, P.A.; Hendrich, S. Urinary disposition of the soybean isoflavones daidzein, genistein and glycitein differs among humans with moderate fecal isoflavone degradation activity. J. Nutr. 1999, 129, 957-962. [CrossRef] [PubMed]

14. Thigpen, J.E.; Setchell, K.D.R.; Ahlmark, K.B.; Locklear, J.; Spahr, T.; Cavines, G.F.; Goelz, M.F.; Haseman, J.K.; Newbold, R.R.; Forsyth, D.B. Phytoestrogen content of purified, open- and closed-formula laboratory animal diets. Lab. Anim. Sci. 1999, 49, 530-536. [PubMed]

15. Kassem, M.A.; Meksem, K.; Iqbal, M.J.; Njiti, V.N.; Banz, W.J.; Winters, T.A.; Wood, A.J.; Lightfoot, D.A. Definition of Soybean Genomic Regions That Control Seed Phytoestrogen Amounts. J. Biomed. Biotechnol. 2004, 2004, 52-60. [CrossRef] 
16. Kassem, M.A.; Shultz, J.; Meksem, K.; Cho, Y.; Wood, A.; Iqbal, M.J.; Lightfoot, D.A. An updated ‘Essex' by ‘Forrest' linkage map and first composite interval map of QTL underlying six soybean traits. Theor. Appl. Genet 2006, 113, 1015-1026. [CrossRef] [PubMed]

17. Gutierrez-Gonzalez, J.J.; Wu, X.; Zhang, J.; Lee, J.-D.; Ellersieck, M.; Shannon, J.G.; Yu, O.; Nguyen, H.T.; Sleper, D.A. Genetic control of soybean seed isoflavone content: Importance of statistical model and epistasis in complex traits. Theor. Appl. Genet. 2009, 119, 1069-1083. [CrossRef]

18. Liang, H.Z.; Yu, Y.L.; Wang, S.F.; Lian, Y.; Wang, T.G.; Wei, T.L.; Gong, P.T.; Liu, X.Y.; Fang, X.J.; Zhang, M.C. QTL Mapping of Isoflavone, Oil and Protein Contents in Soybean (Glycine max L. Merr.). Agric. Sci. China 2010, 9, 1108-1116. [CrossRef]

19. Gutierrez-Gonzalez, J.J.; Vuong, T.D.; Zhong, R.; Yu, O.; Lee, J.-D.; Shannon, G.; Ellersieck, M.; Nguyen, H.T.; Sleper, D.A. Major locus and other novel additive and epistatic loci involved in modulation of isoflavone concentration in soybean seeds. Theor. Appl. Genet. 2011, 123, 1375-1385. [CrossRef]

20. Smallwood, C.J.; Nyinyi, C.N.; Kopsell, D.A.; Sams, C.E.; West, D.R.; Chen, P.; Kantartzi, S.K.; Cregan, P.B.; Hyten, D.L.; Pantalone, V.R. Detection and Confirmation of Quantitative Trait Loci for Soybean Seed Isoflavones. Crop. Sci. 2014, 54, 595-606. [CrossRef]

21. Zhao, G.; Jiang, Z.; Li, D.; Han, Y.; Hu, H.; Wu, L.; Wang, Y.; Gao, Y.; Teng, W.; Li, Y.; et al. Molecular loci associated with seed isoflavone content may underlie resistance to soybean pod borer (Leguminivora glycinivorella). Plant Breed. 2015, 134, 78-84. [CrossRef]

22. Akond, A.; Liu, S.; Kantartzi, S.K.; Meksem, K.; Bellaloui, N.; Lightfoot, D.A.; Yuan, J.; Wang, D.; Anderson, J.; Kassem, M.A. A SNP Genetic Linkage Map Based on the 'Hamilton' by ‘Spencer' Recombinant Inbred Line (RIL) Population of Soybean [Glycine $\max ($ L.) Merr.] Identified QTL for Seed Isoflavone Contents. Plant Breed. 2015, 134, 580-588. [CrossRef]

23. Watanabe, S.; Yamada, R.; Kanetake, H.; Kaga, A.; Anai, T. Identification and characterization of a major QTL underlying soybean isoflavone malonylglycitin content. Breed. Sci. 2019, 69, 564-572. [CrossRef] [PubMed]

24. Han, Y.; Teng, W.; Wang, Y.; Zhao, X.; Wu, L.; Li, D.; Li, W. Unconditional and conditional QTL underlying the genetic interrelationships between soybean seed isoflavone, and protein or oil contents. Plant Breed. 2015, 134, 300-309. [CrossRef]

25. Murphy, S.E.; Lee, E.A.; Woodrow, L.; Seguin, P.; Kumar, J.; Rajcan, I.; Ablett, G.R. Genotype $\times$ Environment Interaction and Stability for Isoflavone Content in Soybean. Crop. Sci. 2009, 49, 1313-1321. [CrossRef]

26. Zhang, H.J.; Li, J.W.; Liu, Y.J.; Jiang, W.Z.; Du, X.L.; Li, L.; Li, X.W.; Su, L.T.; Wang, Q.Y.; Wang, Y. Quantitative trait loci analysis of individual and total isoflavone contents in soybean seeds. J. Genet. 2014, 93, 331-338. [CrossRef] [PubMed]

27. Li, X.; Kamala, S.; Tian, R.; Du, H.; Li, W.; Kong, Y.; Zhang, C. Identification and validation of quantitative trait loci controlling seed isoflavone content across multiple environments and backgrounds in soybean. Mol. Breed. 2018, 38, 8. [CrossRef]

28. Wu, D.; Li, D.; Zhao, X.; Zhan, Y.; Teng, W.; Qiu, L.; Zheng, H.; Li, W.; Han, Y. Identification of a candidate gene associated with isoflavone content in soybean seeds using genome-wide association and linkage mapping. Plant J. 2020, 104, 950-963. [CrossRef]

29. Brown, A.V.; Conners, S.I.; Huang, W.; Wilkey, A.P.; Grant, D.; Weeks, N.T.; Cannon, S.B.; Graham, M.A.; Nelson, R.T. A new decade and new data at SoyBase, the USDA-ARS soybean genetics and genomics database. Nucleic Acids Res. 2020, 49, D1496-D1501. [CrossRef]

30. Kassem, M.A. Two Decades of QTL Mapping of Isoflavone in Soybean Seed. In Soybean Seed Composition: Protein, Oil, Fatty Acids, Amino Acids, Sugars, Mineral Nutrients, and Isoflavone, 1st ed.; Kassem, M.A., Ed.; Springer Nature: Basingstoke, UK, 2021.

31. Grant, D.; Nelson, R.T.; Cannon, S.B.; Shoemaker, R.C. SoyBase, the USDA-ARS soybean genetics and genomics database. Nucleic Acids Res. 2010, 38, D843-D846. [CrossRef]

32. Yang, C.; Yan, J.; Jiang, S.; Li, X.; Min, H.; Wang, X.; Hao, D. Resequencing 250 Soybean Accessions: New Insights into Genes Associated with Agronomic Traits and Genetic Networks. bioRxiv 2021. [CrossRef]

33. Akond, M.; Liu, S.; Schoener, L.; Anderson, J.A.; Kantartzi, S.K.; Meksem, K.; Song, Q.; Wang, D.; Wen, Z.; Lightfoot, D.A.; et al. A SNP-Based Genetic Linkage Map of Soybean Using the SoyS-NP6K Illumina Infinium BeadChip Genotyping Array. Plant Genet. Genom. Sci. 2013, 1, 80-89. [CrossRef]

34. Kassem, M.A.; Ramos, L.; Leandro, L.; Mbofung, G.; Hyten, D.L.; Kantartzi, S.K.; Grier, R.L.; Njiti, V.N.; Cianzio, S.; Meksem, K. The 'PI 438489B' by 'Hamilton' SNP-Based Genetic Linkage Map of Soybean [Glycine max (L.) Merr.] Identified Quantitative Trait Loci that Underlie Seedling SDS Resistance. J. Plant Genome Sci. 2012, 1, 18-30. [CrossRef]

35. Azam, M.; Zhang, S.; Abdelghany, A.M.; Shaibu, A.S.; Feng, Y.; Li, Y.; Tian, Y.; Hong, H.; Li, B.; Sun, J. Seed isoflavone profiling of 1168 soybean accessions from major growing ecoregions in China. Food Res. Int. 2020, 130, 108957. [CrossRef]

36. Hsiao, Y.-H.; Ho, C.-T.; Pan, M.-H. Bioavailability and health benefits of major isoflavone aglycones and their metabolites. J. Funct. Foods 2020, 74, 104164. [CrossRef]

37. Zhang, J.; Ge, Y.; Han, F.; Li, B.; Yan, S.; Sun, J.; Wang, L. Isoflavone Content of Soybean Cultivars from Maturity Group 0 to VI Grown in Northern and Southern China. J. Am. Oil Chem. Soc. 2014, 91, 1019-1028. [CrossRef]

38. Wang, X.; Liu, S.; Yin, X.; Bellaloui, N.; McClure, M.A.; Mengistu, A. Soybean seed isoflavones respond differentially to phosphorus applications in low and high phosphorus soils. Nutr. Cycl. Agroecosyst. 2019, 113, 217-230. [CrossRef]

39. Wang, S.; Basten, C.J.; Zeng, Z.B. Windows QTL Cartographer 2.5; Department of Statistics, NCSU: Raleigh, NC, USA, 2012; Available online: http:/ / statgen.ncsu.edu/qtlcart/WQTLCart.htm (accessed on 10 March 2021).

40. Pei, R.; Zhang, J.; Tian, L.; Zhang, S.; Han, F.; Yan, S.; Wang, L.; Li, B.; Sun, J. Identification of novel QTL associated with soy-bean isoflavone content. Crop. J. 2018, 6, 244-252. [CrossRef] 
41. Schmutz, J.; Cannon, S.B.; Schlueter, J.; Ma, J.; Mitros, T.; Nelson, W.; Hyten, D.L.; Song, Q.; Thelen, J.J.; Cheng, J.; et al. Genome sequence of the palaeopolyploid soybean. Nature 2010, 463, 178-183. [CrossRef] [PubMed]

42. Fliegmann, J.; Furtwängler, K.; Malterer, G.; Cantarello, C.; Schüler, G.; Ebel, J.; Mithöfer, A. Flavone synthase II (CYP93B16) from soybean (Glycine max L.). Phytochemistry 2010, 71, 508-514. [CrossRef] [PubMed]

43. Meng, F.L.; Han, Y.P.; Teng, W.L.; Li, Y.G.; Bin Li, W. QTL underlying the resistance to soybean aphid (Aphis glycines Matsumura) through isoflavone-mediated antibiosis in soybean cultivar 'Zhongdou 27'. Theor. Appl. Genet. 2011, 123, 1459-1465. [CrossRef] [PubMed]

44. Kassem, M.A. QTL that Control Seed Protein, Oil, and Fatty Acids Contents. In Soybean Seed Composition: Protein, Oil, Fatty Acids, Amino Acids, Sugars, Mineral Nutrients, and Isoflavone, 1st ed.; Kassem, M.A., Ed.; Springer Nature: Basingstoke, UK, 2021.

45. Phytozome. Available online: https:// phytozome.jgi.doe.gov/pz/portal.html\# (accessed on 5 July 2021).

46. Hartwig, E.E.; Epps, J.M. Registration of 'Forrest' soybeans. Crop. Sci. 1973, 13, 287. [CrossRef]

47. Bernard, R.L.; Cremeens, C.R. Registration of Williams 82 soybean. Crop. Sci. 1988, 28, 1027-1028. [CrossRef]

48. Shultz, J.L.; Kurunam, D.; Shopinski, K.; Iqbal, M.J.; Kazi, S.; Zobrist, K.; Bashir, R.; Yaegashi, S.; Lavu, N.; Afzal, A.J.; et al. The soybean genome database (SoyGD): A browser for display of duplicated, polyploid, regions and sequence tagged sites on the integrated physical and genetic maps of Glycine max. Nucleic Acids Res. 2006, 34, D758-D765. [CrossRef]

49. Wu, X.; Vuong, T.D.; Leroy, J.A.; Shannon, J.G.; Sleper, D.A.; Nguyen, H.T. Selection of a core set of RILs from Forrest $\times$ Williams 82 to develop a framework map in soybean. Theor. Appl. Genet. 2011, 122, 1179-1187. [CrossRef]

50. Weather Spark. Available online: https://weatherspark.com (accessed on 5 July 2021).

51. Bellaloui, N.; Mengistu, A.; Fisher, D.K.; Abel, C.A. Soybean Seed Composition Constituents as Affected by Drought andPhomopsisin Phomopsis Susceptible and Resistant Genotypes. J. Crop. Improv. 2012, 26, 428-453. [CrossRef]

52. Vuong, T.D.; Sleper, D.A.; Shannon, J.G.; Nguyen, H.T. Novel quantitative trait loci for broad-based resistance to soybean cyst nematode (Heterodera glycines Ichinohe) in soybean PI 567516C. Theor. Appl. Genet. 2010, 121, 1253-1266. [CrossRef]

53. Song, Q.; Yan, L.; Quigley, C.; Fickus, E.; Wei, H.; Chen, L.; Dong, F.; Araya, S.; Liu, J.; Hyten, D.; et al. Soybean BARCSoySNP6K: An assay for soybean genetics and breeding research. Plant J. 2020, 104, 800-811. [CrossRef] [PubMed]

54. Van Ooijen, J.W. Joinmap 4.0 Software for the Calculation of Genetic Linkage Maps in Experimental Populations; Plant Res Intl.: Wageningen, The Netherlands, 2006.

55. Pilet-Nayel, M.L.; Muehlbauer, F.J.; McGee, R.J.; Kraft, J.M.; Baranger, A.; Coyne, C.J. Quantitative trait loci for partial resistance to Aphanomyces root rot in pea. Theor. Appl. Genet. 2002, 106, 28-39. [CrossRef] [PubMed]

56. R Software. Available online: https:/ / www.r-project.org (accessed on 10 March 2021).

57. Voorrips, R.E. MapChart: Software for the graphical presentation of linkage maps and QTL. J. Heredity 2002, 93, 77-78. [CrossRef]

58. Lakhssassi, N.; Lopes-Caitar, V.S.; Knizia, D.; Cullen, M.A.; Badad, O.; El Baze, A.; Zhou, Z.; Embaby, M.G.; Meksem, J.; Lakhssassi, A.; et al. TILLING-by-Sequencing ${ }^{+}$Reveals the Role of Novel Fatty Acid Desaturases (GmFAD2-2s) in Increasing Soybean Seed Oleic Acid Content. Cells 2021, 10, 1245. [CrossRef] [PubMed]

59. Bolger, A.M.; Lohse, M.; Usadel, B. Trimmomatic: A flexible trimmer for Illumina sequence data. Bioinformatics 2014, 30, 2114-2120. [CrossRef] [PubMed]

60. Dobin, A.; Gingeras, T.R. Mapping RNA-seq Reads with STAR. Curr. Protoc. Bioinform. 2015, 51, 11.14.1-11.14.19. [CrossRef] [PubMed]

61. Trapnell, C.; Pachter, L.; Salzberg, S.L. TopHat: Discovering splice junctions with RNA-Seq. Bioinformatics 2009, $25,1105-1111$. [CrossRef]

62. Anders, S.; Pyl, P.T.; Huber, W. HTSeq-A Python framework to work with high-throughput sequencing data. Bioinformatics 2015, 31, 166-169. [CrossRef] [PubMed]

63. Love, M.I.; Huber, W.; Anders, S. Moderated estimation of fold change and dispersion for RNA-seq data with DESeq2. Genome Biol. 2014, 15, 550. [CrossRef]

64. Lakhssassi, N.; Zhou, Z.; Liu, S.; Piya, S.; Patil, G.B.; Cullen, M.A.; El Baz, A.; Badad, O.; Embaby, M.G.; Meksem, J.; et al. Soybean TILLING-by-Sequencing+ reveals the role of novel GmSACPD members in the unsaturated fatty acid biosynthesis while maintaining healthy nodules. J. Exp. Bot. 2020, 71, 6969-6987. [CrossRef] [PubMed] 